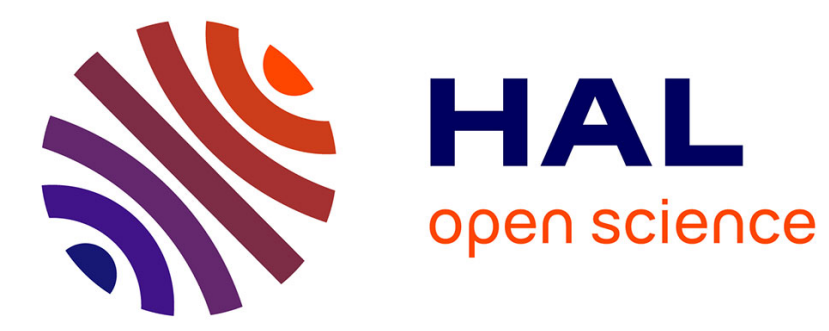

\title{
Nonlinear and multiphysics evaluation of residual stresses in coils
}

Daniel Weisz-Patrault

\section{To cite this version:}

Daniel Weisz-Patrault. Nonlinear and multiphysics evaluation of residual stresses in coils. Applied Mathematical Modelling, 2018. hal-01823186

\section{HAL Id: hal-01823186 https://hal.science/hal-01823186}

Submitted on 25 Jun 2018

HAL is a multi-disciplinary open access archive for the deposit and dissemination of scientific research documents, whether they are published or not. The documents may come from teaching and research institutions in France or abroad, or from public or private research centers.
L'archive ouverte pluridisciplinaire HAL, est destinée au dépôt et à la diffusion de documents scientifiques de niveau recherche, publiés ou non, émanant des établissements d'enseignement et de recherche français ou étrangers, des laboratoires publics ou privés. 


\title{
Nonlinear and multiphysics evaluation of residual stresses in coils
}

\author{
Daniel Weisz-Patrault \\ LMS, École Polytechnique, CNRS, Université Paris-Saclay, 91128 Palaiseau, France
}

\begin{abstract}
This article deals with residual stress evaluation within the framework of numerical simulation of the coiling process of steel. Plastic deformations along with multiphase transitions are responsible for large irreversible strain leading to major residual stress issues. A nonlinear mixed analytical/numerical approach is developed to compute residual stresses generated by different contributions of inelastic strain occurring during the coiling process (including both winding and cooling). In particular, transformation-induced plasticity is taken into account. Contact stresses at the interfaces between layers are updated at each time step by a minimization procedure. The analytical part of the proposed strategy is validated by comparison with a finite element computation. Then a coil composed of 196 layers is considered under typical industrial conditions. Numerical results are discussed to present the industrial application of the proposed approach.
\end{abstract}

Keywords: Residual stress, Multiphysics, Contact problem, Coiling process

\section{Introduction}

The current dynamic of steel manufacturing is to regularly develop new stronger grades enabling users to reduce strip thicknesses and thus reduce produced tonnages, which results in energy efficiency by minimizing, for instance, the total mass of vehicles. One of the major issues related to this evolution of steel production is the forming processes (see Figure 1) that lead to serious residual stress problems, which in turn can result in instabilities such as local buckling during the rolling process [1]. This contribution focuses on the coiling process, which consists in winding a strip on itself for storage. Within this framework, residual stress issues can result in local buckling or coil sagging, as demonstrated in Figure 2. Other defects are detailed in [2]. When the inner mandrel is removed from the coil, residual stresses may be sufficiently compressive to cause buckling of some layers. Furthermore, the overall inelastic strain due to thermal expansion and phase transitions may be responsible for large displacements leading to coil sagging. In this article I attempt to quantify the overall inelastic strain as well as the associated residual stress by modeling the whole coiling process. The outcomes are necessary for the prediction of some defects such as coil sagging even though additional work is still needed.

Residual stresses generated during this process have been studied, for instance, by Quach et al. [3, 4], who developed a closed-form analytical solution of a pure bending problem (i.e., disregarding the effect of contacts). The contribution of several successive processes was considered by Moen et al. [5] also for a pure bending problem. However, contact pressures are heterogeneous along the coil axis, which results in significant variations of residual stresses. Contact pressures can concentrate near the central axial position and significantly contribute to the formation of plastic deformations and therefore to the final residual stress field. Several coiling models that take into account contacts have been proposed. One can mention purely elastic models developed by Edwards and Boulton [2], Hudzia et al. [6], and Kedl [7], where the radial Young's modulus is set as a function of contact pressure to model roughness of contacts. One can also mention a fast simplified coil winding model based on the Airy function proposed by Hinton et al. $[8,9]$. However, the coiling process consists not only in winding the strip around a mandrel but also in cooling the coil for several hours after removal of the internal mandrel. Thus, contact pressures are completely redistributed and thermal expansion and phase transitions occur. Previous studies dealt only with the isothermal winding phase of the process and ignored the cooling phase. However, it will be demonstrated here that residual stresses are significantly modified during the cooling phase. 


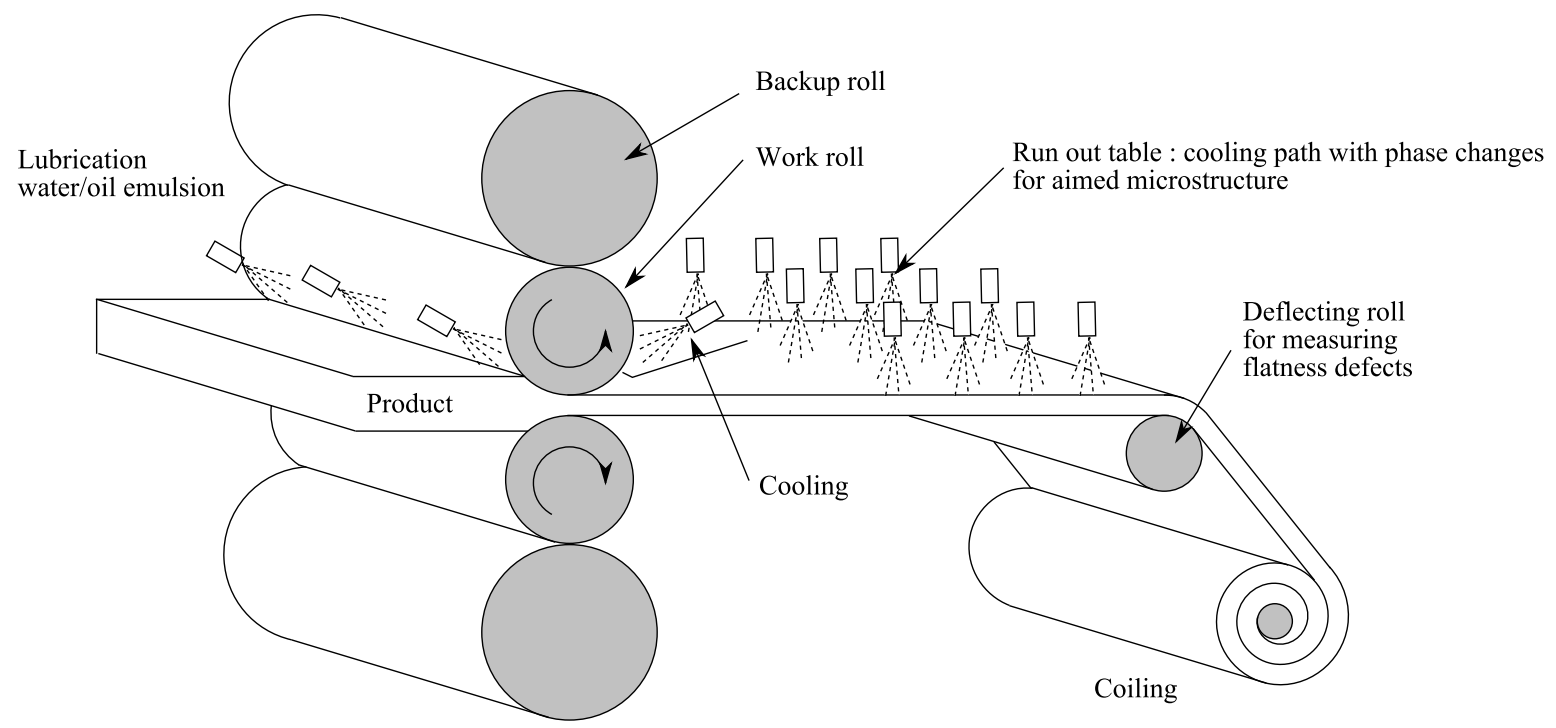

Figure 1: Forming processes

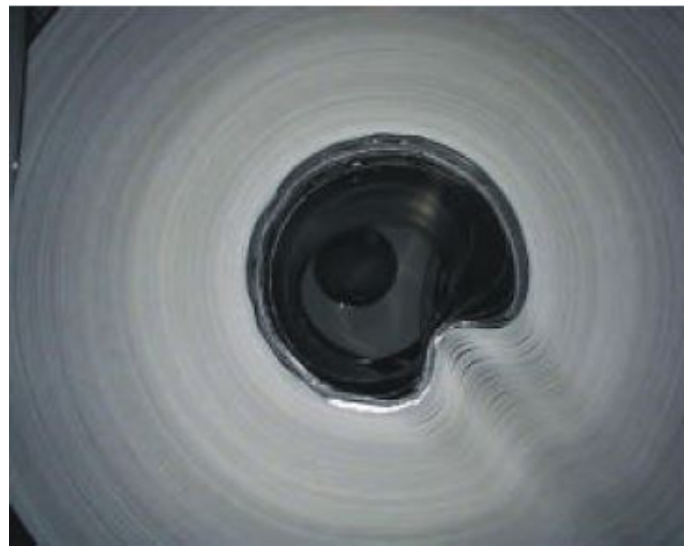

(a) Local buckling

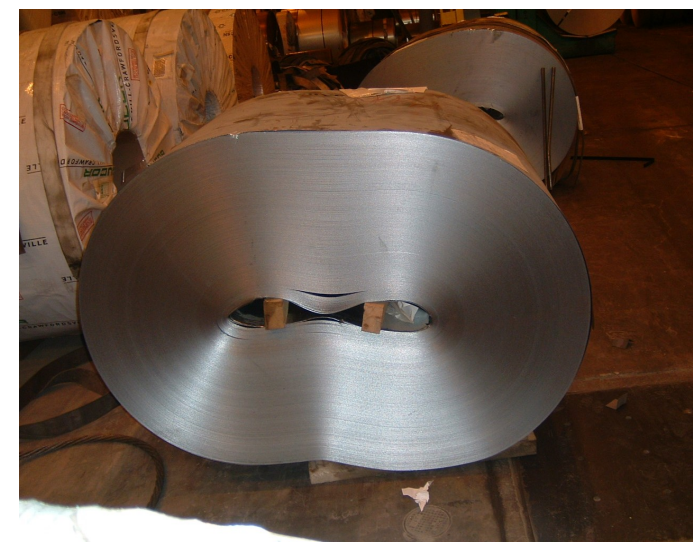

(b) Coil sagging

Figure 2: Instabilities

Recently developed grades undergo significant phase transitions when the coil cools down. Additional inelastic strain occurs because of thermal expansion, phase density mismatch, and transformation-induced plasticity. Thus, this article contributes to the development of a global numerical strategy that solves the nonlinear and coupled problem of a strip winding on itself and then cooling by undergoing phase transitions. Couplings between thermal sciences, metallurgy, and solid mechanics should be considered as well as several types of nonlinearity, such as finite strain computations, elastic-plastic behavior, and contact stress evaluation during the winding phase of the process, and temperature-dependent heat sources because of enthalpy changes and transformation-induced plasticity when the coil cools down.

This article relies on previous contributions. Weisz-Patrault et al. [10] proposed a nonlinear isothermal elasticplastic model of the winding phase accounting for roughness of contacts and finite strain to consider large rotations. This model was based on previous work by Weisz-Patrault et al. [11, 12]. Then Weisz-Patrault [13] developed a solution for the cooling phase by coupling heat conduction and multiphase transition problems accounting for thermal contact resistance. The present article aims to establish a numerical strategy to compute residual stresses on the basis of the previously developed models. Each layer of the coil is subjected at each time step to an eigenstrain increment composed of several contributions, namely:

(i) the thermometallurgical hydrostatic strain due to thermal expansion and density mismatch between different 
phases;

(ii) the transformation-induced plastic strain;

(iii) classical plastic strain due to temperature variations.

The evaluation of the eigenstrain increment necessitates the computation of temperature and phase proportion rates that are obtained through the method proposed by Weisz-Patrault [13]. Furthermore, the macroscopic stress tensor is also needed for the computation of the eigenstrain increment of the following time step. Thus, the residual stress field should be computed at each time step as an input of the following time step, and one cannot jump directly to the last time step by simply integrating the time-dependent eigenstrain. For the exact same reason, contact stresses are also updated at each time step by use of minimization procedures as detailed in Section 7 . The general modeling structure is presented in Figure 3.

Macroscopic plasticity is not considered in this contribution; that is, that the macroscopic equivalent stress does not reach the macroscopic yield stress. Although macroscopic yield stress is not reached, certain phases (e.g., austenite) can locally undergo plastic deformations. This local plasticity is taken into account in the eigenstrain obtained from the homogenization procedure proposed by Weisz-Patrault [14]. The nonlinear part is therefore limited in this article to the imposed eigenstrain, which should be computed incrementally, and to the contact stress evaluation. Thus, the present work consists in computing all contributions of the overall eigenstrain. Then an analytical solution of the elastic problem subjected to the eigenstrain is sought for each layer of the coil and at each time step. In addition, contact stresses at each interface between layers are also updated at each time step by use of a numerical minimization procedure relying on contact laws accounting for roughness, following the ideas of Sutcliffe [15] or Sheu and Wilson [16].

Mathematical developments rely, on the one hand, on a series expansion with respect to a certain function sequence and, on the other hand, on Papkovich-Neuber potential theory involving harmonic and biharmonic functions as presented for, instance, by Barber [17]. One can also mention a very elegant potential theory mainly developed by Muskhelishvili [18] applicable for plane elasticity and relying on complex potentials. An extension in quaternionic algebra dedicated to three-dimensional elasticity was proposed by Weisz-Patrault et al. [19], but is not used in this contribution for simplicity. 


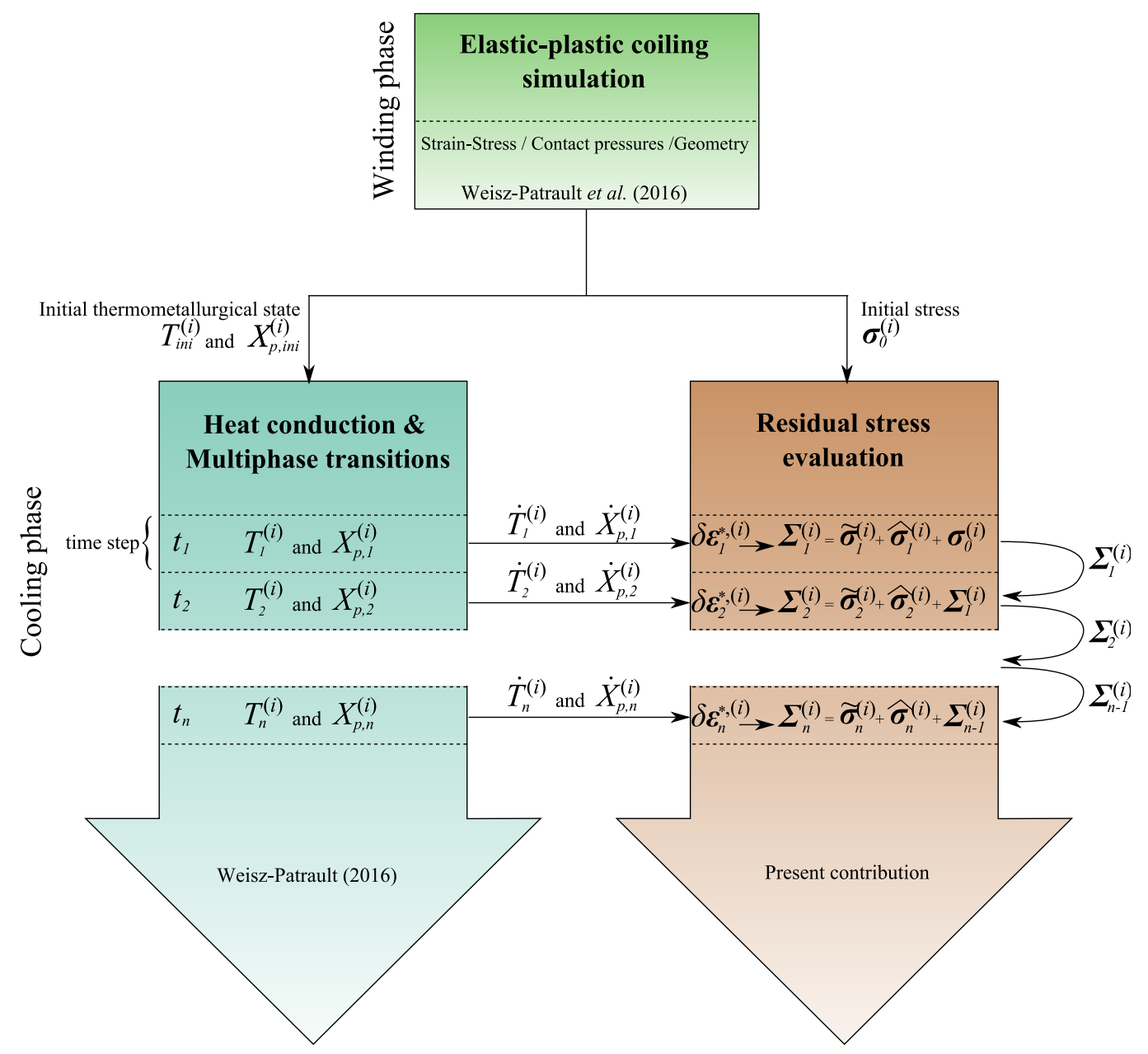

Figure 3: General modeling structure

The main outcome of the proposed approach is the computation of residual stress and inelastic strain in the coil. The work of Weisz-Patrault et al. [10] and Weisz-Patrault [13] and the present article contribute to the development of an effective tool that enables users to perform parametric studies, especially for recent grades for which residual stress issues arise frequently. To the best of my knowledge, this article presents for the first time a complete approach enabling users to track irreversible strains and residual stresses through the whole coiling process, including the winding phase and the cooling phase. The situation before the coiling process is not considered in this contribution, and can be directly measured through an optical or mechanical measurement system as reviewed by Molleda et al. [20]. An inverse method based on conformal mapping techniques was proposed by Weisz-Patrault [21] to evaluate residual stresses by deflection of the steel strip on a hollow cylinder.

\section{General equations and notation}

This section details the global equation system that is solved at each time step (denoted by $n$ ). The main notation is summarized in Figure 4. The coil is constituted of $N$ layers. Some assumptions are needed to obtain a convenient analytical solution. Each layer (denoted by $(i)$, where $1 \leq i \leq N$ ) is characterized at each time step $n$ by lower and upper surface radii denoted, respectively, by $R_{i n f, n}^{(i)}(z)$ and $R_{\text {sup }, n}^{(i)}(z)$ (where $r$ and $z$ denote the cylindrical coordinates). They clearly depend on the axial position $z$. However, the following analytical solution relies on $z$-independent lower and upper surface radii, denoted by $r_{i n f, n}^{(i)}$ and $r_{s u p, n}^{(i)}$, defined as the average values of $R_{\text {inf, },}^{(i)}(z)$ and $R_{\text {sup }, n}^{(i)}(z)$ over the coil width:

$$
r_{i n f, n}^{(i)}=\frac{1}{2 L} \int_{-L}^{L} R_{i n f, n}^{(i)}(z) \mathrm{d} z \quad \text { and } \quad r_{\text {sup }, n}^{(i)}=\frac{1}{2 L} \int_{-L}^{L} R_{\text {sup }, n}^{(i)}(z) \mathrm{d} z
$$


where $L$ denotes the half width. Thus, each layer is described by

$$
\Omega_{n}^{(i)}=\left\{(r, z) \in\left[r_{i n f, n}^{(i)}, r_{s u p, n}^{(i)}\right] \times[-L, L]\right\}
$$

In addition, $T_{n}^{(i)}(r, z)$ denotes the temperature field in the $i$ th layer at the current time step $n$. In this article a multiphase mixture is considered. Phase proportions are denoted by $X_{p, n}^{(i)}$ (with $1 \leq p \leq N_{p}$, where $N_{p}$ is the number of phases). Thus, Lamé's coefficients $\left(\lambda_{p, n}^{(i)}\left(T^{(i)}\right), \mu_{p, n}^{(i)}\left(T^{(i)}\right)\right)$ are temperature dependent and therefore inhomogeneous along both the radial direction and the axial direction. The analytical solution relies on constant material properties in each layer $\left(\lambda_{p, n}^{(i)}, \mu_{p, n}^{(i)}\right)$, which are computed as follows:

$$
\left\{\begin{array}{l}
\lambda_{p, n}^{(i)}=\frac{1}{2 L\left(r_{\text {sup }, n}^{(i)}-r_{i n f, n}^{(i)}\right)} \int_{r_{i n f, n}^{(i)}}^{r_{s u p, n}^{(i)}} \int_{-L}^{L} \lambda_{p, n}^{(i)}\left(T_{n}^{(i)}(r, z)\right) \mathrm{d} r \mathrm{~d} z \\
\mu_{p, n}^{(i)}=\frac{1}{2 L\left(r_{\text {sup }, n}^{(i)}-r_{i n f, n}^{(i)}\right)} \int_{r_{i n f, n}^{(i)}}^{(i)} \int_{-L}^{L} \mu_{p, n}^{(i)}\left(T_{n}^{(i)}(r, z)\right) \mathrm{d} r \mathrm{~d} z
\end{array}\right.
$$

In addition, Lamé's coefficients of the multiphase mixture $\left(\lambda_{n}^{(i)}, \mu_{n}^{(i)}\right)$ are computed as follows:

$$
\lambda_{n}^{(i)}=\sum_{p=1}^{N_{p}} X_{p, n}^{(i)} \lambda_{p, n}^{(i)} \quad \text { and } \quad \mu_{n}^{(i)}=\sum_{p=1}^{N} X_{p, n}^{(i)} \mu_{p, n}^{(i)}
$$

At each time step, an additional eigenstrain increment $\delta \varepsilon_{n}^{*(i)}$ is imposed. Thus, an additional elastic problem should be solved so as to update the previous mechanical state. The global additional problem is written as follows:

$$
\forall 1 \leq i \leq N, \begin{cases}\operatorname{div}\left[\sigma_{n}^{(i)}\right]=0 & \text { (equilibrium) } \\ \boldsymbol{\sigma}_{n}^{(i)}=\lambda_{n}^{(i)} \operatorname{tr}\left[\boldsymbol{\varepsilon}_{n}^{e,(i)}\right] \boldsymbol{I}+2 \mu_{n}^{(i)} \boldsymbol{\varepsilon}_{n}^{e,(i)} & \text { (isotropic behavior) } \\ \boldsymbol{\varepsilon}_{n}^{(i)}=\frac{1}{2}\left(\boldsymbol{\nabla}\left[\boldsymbol{u}_{n}^{(i)}\right]+\boldsymbol{\nabla}\left[\boldsymbol{u}_{n}^{(i)}\right]^{T}\right) & \text { (compatibility) } \\ \boldsymbol{\varepsilon}_{n}^{e,(i)}=\boldsymbol{\varepsilon}_{n}^{(i)}-\delta \boldsymbol{\varepsilon}_{n}^{*,(i)} & \text { (elastic strain tensor) }\end{cases}
$$

where $\boldsymbol{\sigma}_{n}^{(i)}, \boldsymbol{\varepsilon}_{n}^{(i)}$, and $\boldsymbol{u}_{n}^{(i)}$ denote the additional stress and strain tensors and the additional displacement vector, respectively. The total stress accumulated from all the previous time steps is denoted by

$$
\boldsymbol{\Sigma}_{n-1}^{(i)}=\sum_{l=0}^{n-1} \sigma_{l}^{(i)}
$$

where $\sigma_{0}^{(i)}$ is the initial stress tensor from previous processes as detailed in Figure 3. Furthermore, the eigenstrain increment $\delta \boldsymbol{\varepsilon}_{n}^{*,(i)}$ depends on $\boldsymbol{\Sigma}_{n-1}^{(i)}$ as detailed in Section 3. It is decomposed into different contributions:

$$
\delta \varepsilon_{n}^{*,(i)}=\delta \varepsilon_{n}^{t h m,(i)}+\delta \varepsilon_{n}^{t p,(i)}+\delta \varepsilon_{n}^{c p,(i)}
$$

where $\delta \boldsymbol{\varepsilon}_{n}^{\text {thm,(i) }}$ represents the thermometallurgical strain increment, which corresponds to volume variations due to thermal expansion and density mismatch between phases, $\delta \varepsilon_{n}^{t p,(i)}$ is the strain increment due to transformationinduced plasticity, and $\delta \varepsilon_{n}^{c p,(i)}$ is the classical plastic strain increment induced by temperature variations in the multiphase mixture. Classical plasticity induced by stress variations can also be considered but it is usually negligible.

In addition, the boundary conditions for the additional problem (5) are

$$
\forall 1 \leq i \leq N,\left\{\begin{array}{l}
\boldsymbol{\sigma}_{n}^{(i)}\left(r_{\text {sup }, n}^{(i)}, z\right) \cdot \boldsymbol{n}\left(r_{\text {sup }, n}^{(i)}, z\right)=\boldsymbol{T}_{\text {sup }, n}^{(i)}(z) \\
\boldsymbol{\sigma}_{n}^{(i)}\left(r_{\text {inf }, n}^{(i)}, z\right) \cdot \boldsymbol{n}\left(r_{i n f, n}^{(i)}, z\right)=\boldsymbol{T}_{\text {inf }, n}^{(i)}(z) \\
\boldsymbol{\sigma}_{n}^{(i)}(r, \pm L) \cdot \boldsymbol{n}(r, \pm L)=0 \\
\boldsymbol{T}_{\text {inf, }, n}^{(i+1)}(z)=-\boldsymbol{T}_{\text {sup }, n}^{(i)}(z) \\
\boldsymbol{T}_{\text {sup }, n}^{(N)}(z)=\boldsymbol{T}_{\text {inf }, n}^{(1)}(z)=0
\end{array}\right.
$$


where $\boldsymbol{n}$ is the unit normal vector and $\boldsymbol{T}_{\text {sup,n }}^{(i)}(z)$ and $\boldsymbol{T}_{\text {inf,n }}^{(i)}(z)$ denote the additional traction (at $r=r_{\text {sup }, n}^{(i)}$ and $r=r_{i n f, n}^{(i)}$, respectively), decomposed as follows:

$$
\forall 1 \leq i \leq N,\left\{\begin{array}{l}
\boldsymbol{T}_{s u p, n}^{(i)}(z)=-P_{\text {sup }, n}^{(i)}(z) \boldsymbol{e}_{r}+S_{\text {sup }, n}^{(i)}(z) \boldsymbol{e}_{z} \\
\boldsymbol{T}_{\text {inf }, n}^{(i)}(z)=P_{\text {inf }, n}^{(i)}(z) \boldsymbol{e}_{r}+S_{\text {inf }, n}^{(i)}(z) \boldsymbol{e}_{z}
\end{array}\right.
$$

where $P_{\text {sup }, n}^{(i)}(z)=P_{\text {inf,n }}^{(i+1)}(z)$ are the additional contact pressures and $S_{\text {sup }, n}^{(i)}(z)=-S_{\text {inf,n }}^{(i+1)}(z)$ are the additional contact shear stresses that are evaluated by numerical minimization as detailed in Section 7.

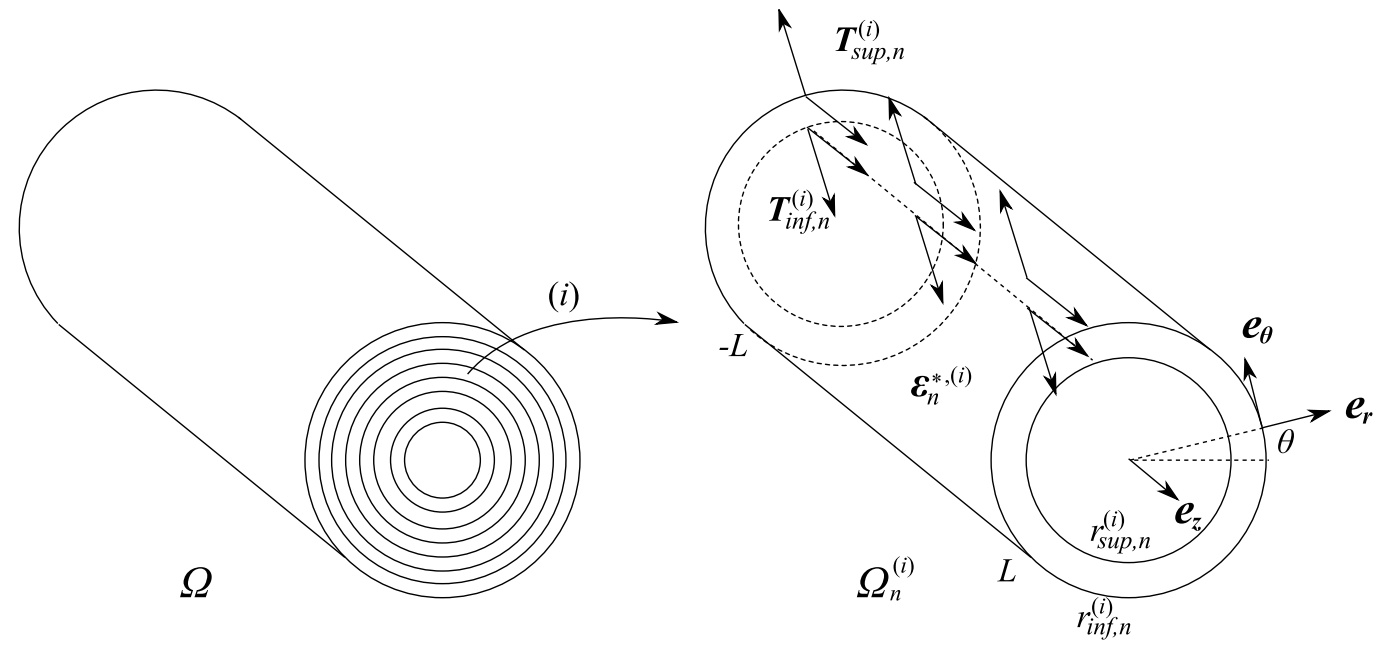

Figure 4: Global system and notation

\section{Eigenstrain}

This section is dedicated to the computation (for each layer $(i)$ and at the current time step $n$ ) of the eigenstrain increment (7) that contains most of the nonlinear information (along with contact stress evaluation). In all the following, the superscript $(i)$ and the time step index $n$ are omitted for readability. For instance, the displacement vector $\boldsymbol{u}_{n}^{(i)}$ is denoted by $\boldsymbol{u}$ in the rest of this article. Transformation-induced plasticity and classical plasticity are computed following the recent theoretical work of Weisz-Patrault [14] that extends the well-known model proposed by Leblond et al. [22]. Multiphase transitions are taken into account, and several assumptions are relaxed. However, expressions are particularized for this study; that is, for cooling only and for the following phase transitions: austenite to ferrite, austenite to pearlite, austenite to bainite, and austenite to martensite. In addition, the following convention is used: $p=1$ for austenite, $p=2$ for ferrite, $p=3$ for pearlite, $p=4$ for bainite, and $p=5$ for martensite. Equations (10)-(24), extracted from [14], are recalled here for clarity. The additional stress $\sigma$ is distributed in different phases as follows:

$$
\sigma_{p}=\frac{\widetilde{E}_{p}}{\widetilde{E}} \sigma
$$

where $\sigma_{p}$ is the additional stress in phase $p$ and

$$
\left\{\begin{array}{l}
\widetilde{E}=\sum_{p \in \mathcal{E}} X_{p} E_{p}+\sum_{p \in \mathcal{P}} X_{p} \gamma_{p} \sigma_{p}^{Y} \\
\widetilde{E}_{p}=\left\{\begin{array}{lll}
E_{p} & \text { if } & p \in \mathcal{E} \\
\gamma_{p} \sigma_{p}^{Y} & \text { if } & p \in \mathcal{P}
\end{array}\right.
\end{array}\right.
$$

where for each phase $p, \sigma_{p}^{Y}$ is the yield stress, $E_{p}$ is the Young's modulus, and $\gamma_{p}$ is the hardening parameter. In addition, $\mathcal{E} \subset\left\{1, \cdots, N_{p}\right\}$ is the index set of phases that do not reach the plastic regime and $\mathcal{P} \subset\left\{1, \cdots, N_{p}\right\}$ 
is the index set of phases that reach the plastic regime. The same expression as (10) holds for the total stress accumulated from all the previous time steps $\boldsymbol{\Sigma}$ defined by (6):

$$
\boldsymbol{\Sigma}_{p}=\frac{\widetilde{E}_{p}}{\widetilde{E}} \boldsymbol{\Sigma}
$$

The eigenstrain increment is obtained as follows:

$$
\delta \boldsymbol{\varepsilon}^{*}=\dot{\boldsymbol{\varepsilon}}^{*} \delta t
$$

where $\delta t$ is the time increment. The hydrostatic thermometallurgical strain rate is shown to be

$$
\dot{\boldsymbol{\varepsilon}}^{t h m}=\sum_{p=2}^{N_{p}} \frac{1}{3}\left(\frac{\rho_{1}(T)}{\rho_{p}(T)}-1\right) \dot{X}_{p}+\left(\alpha_{1}+\sum_{p=2}^{N_{p}} X_{p}\left(\alpha_{p}-\alpha_{1}\right)\right) \dot{T}
$$

where $\rho_{p}$ and $\alpha_{p}$ are, respectively, the density and the thermal expansion coefficient of phase $p$. Consider $\widetilde{X}$ to be the total product phase proportion:

$$
\widetilde{X}=1-X_{1}=\sum_{p=2}^{N_{p}} X_{p}
$$

And consider $\Delta \sigma^{Y}$ to be the distance between the yield stress and the von Mises equivalent stress in austenite:

$$
\Delta \sigma^{Y}=\sqrt{\left(\sigma_{1}^{Y}\right)^{2}-\left(\Sigma_{1}^{e q}\right)^{2}}
$$

where $\Sigma_{p}^{e q}$ is the von Mises equivalent stress in phase $p$ computed from $\boldsymbol{\Sigma}_{p}$. Consider the following material parameters:

$$
\left\{\begin{array}{l}
\zeta=\frac{\left(3 \lambda_{1}+2 \mu_{1}\right) 2 \mu_{1}}{\lambda_{1}+2 \mu_{1}} \\
\xi_{p}=\frac{2 \mu_{1}\left(9 \lambda_{1}+14 \mu_{1}\right)}{\lambda_{1}\left(9 \mu_{1}+6 \mu_{p}\right)+2 \mu_{1}\left(7 \mu_{1}+8 \mu_{p}\right)}
\end{array}\right.
$$

If $\Sigma_{1}^{e q}<\sigma_{1}^{Y}$, the transformation-induced plastic strain rate is

$$
\dot{\boldsymbol{\varepsilon}}^{t p}=\sum_{p=2}^{N_{p}} \frac{S_{p}}{\sigma_{p}^{Y}} \frac{\sigma_{p}^{Y}-\Sigma_{p}^{e q}}{\mu_{p} \xi_{p}} \dot{X}_{p}+ \begin{cases}0 & \text { if } \frac{\Delta \sigma^{Y}}{\zeta\left|\widetilde{\varepsilon}^{t h m}\right|}>1 \\ -\frac{3\left|\widetilde{\varepsilon}^{h m}\right| S_{1}}{\sigma_{1}^{Y}} \ln \left(\frac{\Delta \sigma^{Y}}{\zeta\left|\widetilde{\varepsilon}^{t h m}\right|}\right) \sum_{p=2}^{N_{p}} \dot{X}_{p} & \text { if } \quad \widetilde{X} \leq \frac{\Delta \sigma^{Y}}{\zeta||^{t h m} \mid} \leq 1 \\ -\frac{3\left|\tilde{\varepsilon}^{h m}\right| S_{1}}{\sigma_{1}^{Y}} \ln (\widetilde{X}) \sum_{p=2}^{N_{p}} \dot{X}_{p} & \text { if } \quad \widetilde{X}>\frac{\Delta \sigma^{Y}}{\zeta\left|\widetilde{\varepsilon}^{t h m}\right|}\end{cases}
$$

where $\boldsymbol{S}_{p}=\boldsymbol{\Sigma}_{p}-\operatorname{tr}\left[\boldsymbol{\Sigma}_{p}\right] \mathbf{1} / 3$ is the total deviatoric stress tensor of phase $p$.

If $\Sigma_{1}^{e q}=\sigma_{1}^{Y}$,

$$
\dot{\boldsymbol{\varepsilon}}^{t p}=\sum_{p=2}^{N_{p}} \frac{\boldsymbol{S}_{p}}{\sigma_{p}^{Y}} \frac{\sigma_{p}^{Y}-\Sigma_{p}^{e q}}{\mu_{p} \xi_{p}} \dot{X}_{p}+\left\{\begin{array}{lll}
0 & \text { if } \quad \tilde{X}<\widetilde{X}_{\min } \\
-\frac{3\left|\widetilde{\varepsilon}^{t h m}\right| \boldsymbol{S}_{1}}{\sigma_{1}^{Y}} \ln (\widetilde{X}) \sum_{\substack{p=2 \\
\dot{X}_{p}>0}}^{N_{p}} \dot{X}_{p} & \text { if } \quad \widetilde{X} \geq \widetilde{X}_{\min }
\end{array}\right.
$$

with the initial value

$$
\varepsilon_{i n i}^{t p}=\frac{2 \widetilde{\varepsilon}^{h m}}{1-\widetilde{X}_{\min }} \widetilde{X}_{\min } \ln \left(\widetilde{X}_{\text {min }}\right)
$$


where $\widetilde{X}_{\text {min }}$ (set to 0.03 in this article) is the minimal phase proportion that can be produced from pure austenite and where $\widetilde{\varepsilon}^{\text {thm }}$ is the hydrostatic eigenstrain generated in all product phases because of temperature variation and phase transitions:

$$
\widetilde{\varepsilon}^{\text {hm }}=\sum_{p=2}^{N_{p}} \frac{X_{p}}{1-X_{1}}\left(\frac{1}{3}\left(\frac{\rho_{1}(T)}{\rho_{p}(T)}-1\right)+\frac{\rho_{1}(T)}{\rho_{p}(T)}\left(\alpha_{p}-\alpha_{1}\right)\left(T-T_{\text {ini }}\right)\right)>0
$$

$\widetilde{\varepsilon}^{\text {Thm }}$ given by (21) is related to the microstructure and should not be confused with the homogenized hydrostatic strain $\boldsymbol{\varepsilon}^{\text {thm }}$ given by (14). If $\Sigma_{1}^{e q}<\sigma_{1}^{Y}$, the classical plastic strain rate due to thermal variations is

$$
\dot{\boldsymbol{\varepsilon}}^{c p}=\left\{\begin{array}{lll}
0 & \text { if } \quad \frac{\Delta \sigma^{Y}}{\zeta\left|\widetilde{\mathcal{E}}^{t h m}\right|}>1 \\
\frac{-3 \widetilde{\alpha} S_{1}}{\sigma_{1}^{Y}} \widetilde{X} \ln \left(\frac{\Delta \sigma^{Y}}{\zeta\left|\widetilde{\mathcal{E}}^{t h m}\right|}\right) \dot{T} & \text { if } & \widetilde{X} \leq \frac{\Delta \sigma^{Y}}{\zeta\left|\widetilde{\mathcal{E}}^{t h m}\right|} \leq 1 \\
\frac{-3 \widetilde{\alpha} S_{1}}{\sigma_{1}^{Y}} \widetilde{X} \ln (\widetilde{X}) \dot{T} & \text { if } \quad \widetilde{X}>\frac{\Delta \sigma^{Y}}{\zeta\left|\widetilde{\varepsilon}^{t h m}\right|}
\end{array}\right.
$$

If $\Sigma_{1}^{e q}=\sigma_{1}^{Y}$,

$$
\dot{\boldsymbol{\varepsilon}}^{c p}=\frac{-3 \widetilde{\alpha} \boldsymbol{S}_{1}}{\sigma_{1}^{Y}} \widetilde{X} \ln (\widetilde{X}) \dot{T}
$$

where $\widetilde{\alpha}$ represents the homogenized difference between the thermal expansion coefficient in the product phases and the thermal expansion coefficient in austenite:

$$
\widetilde{\alpha}=\sum_{p=2}^{N_{p}} \frac{X_{p}}{1-X_{1}}\left(\alpha_{p}-\alpha_{1}\right)<0
$$

\section{Problem decomposition for each layer}

This section deals with the general scheme for establishing an analytical solution. For each layer, boundary conditions are applied to the hollow cylinder, according to (8). The determination of $\boldsymbol{T}_{\text {sup }}(z)$ and $\boldsymbol{T}_{\text {inf }}(z)$ is done by a minimization procedure detailed in Section 7. The additional problem (5) way be rewritten as a function of displacements instead of the stress tensor. Then for each layer it reduces to the inhomogeneous Navier equation

$$
\mu \boldsymbol{\Delta} \boldsymbol{u}+(\lambda+\mu) \boldsymbol{\nabla} \operatorname{div} \boldsymbol{u}=\operatorname{div}\left[\lambda \operatorname{tr}\left[\delta \boldsymbol{\varepsilon}^{*}\right] \boldsymbol{I}+2 \mu \delta \boldsymbol{\varepsilon}^{*}\right]
$$

such that the boundary conditions (8) are verified. The Navier equation (25) is linear. Thus, nonlinearity aspects are related not to the equation itself but rather to the computation of the eigenstrain increment $\delta \varepsilon^{*}$ and the determination of contact stresses $\boldsymbol{T}_{\text {sup }}(z)$ and $\boldsymbol{T}_{\text {inf }}(z)$. Therefore, a general solution of (25) may be written as the linear combination of a particular solution $\widetilde{\boldsymbol{u}}$ and a homogeneous solution $\widehat{\boldsymbol{u}}$. Corresponding stress and stain tensors are denoted accordingly:

$$
\left\{\begin{array}{l}
u=\widetilde{u}+\widehat{u} \\
\sigma=\widetilde{\sigma}+\widehat{\sigma} \\
\varepsilon=\widetilde{\varepsilon}+\widehat{\varepsilon}
\end{array}\right.
$$

The decomposition is summarized in Figure 5. It should be noted that $\widetilde{\boldsymbol{u}}$ may be any particular solution of (27) without consideration of specific boundary conditions:

$$
\mu \Delta \widetilde{\boldsymbol{u}}+(\lambda+\mu) \boldsymbol{\nabla} \operatorname{div} \widetilde{\boldsymbol{u}}=\operatorname{div}\left[\lambda \operatorname{tr}\left[\delta \boldsymbol{\varepsilon}^{*}\right] \boldsymbol{I}+2 \mu \delta \boldsymbol{\varepsilon}^{*}\right]
$$

However, $\widetilde{\boldsymbol{u}}$ is a solution of (28) verifying the boundary conditions (29), so the global boundary conditions (8) are verified:

$$
\begin{gathered}
\mu \Delta \widehat{\boldsymbol{u}}+(\lambda+\mu) \boldsymbol{\nabla} \operatorname{div} \widehat{\boldsymbol{u}}=0 \\
\forall 1 \leq i \leq N,\left\{\begin{array}{l}
\widehat{\boldsymbol{\sigma}}\left(r_{\text {sup }}, z\right) \cdot \boldsymbol{n}\left(r_{\text {sup }}, z\right)=-\widetilde{\boldsymbol{\sigma}}\left(r_{\text {sup }}, z\right) \cdot \boldsymbol{n}\left(r_{\text {sup }}, z\right)+\boldsymbol{T}_{\text {sup }}(z) \\
\widehat{\boldsymbol{\sigma}}\left(r_{\text {inf }}, z\right) \cdot \boldsymbol{n}\left(r_{\text {inf }}, z\right)=-\widetilde{\boldsymbol{\sigma}}\left(r_{\text {inf }}, z\right) \cdot \boldsymbol{n}\left(r_{\text {inf }}, z\right)+\boldsymbol{T}_{\text {inf }}(z) \\
\boldsymbol{\sigma}(r, \pm L) \cdot \boldsymbol{n}(r, \pm L)=0
\end{array}\right.
\end{gathered}
$$


$\boldsymbol{T}_{\text {sup }}(z)$ and $\boldsymbol{T}_{\text {inf }}(z)$ are arbitrary in Sections 5 and 6. Correct values are determined by numerical minimization as detailed in Section 7.

In addition, the third boundary condition at $z= \pm L$ in (29) presents more difficulties with respect to the analytical treatment. For simplicity, this edge condition is verified not locally but in a weaker sense; that is, the resultant force of surface traction vanishes at the edges. Moreover, since the analytical development relies on a potential formulation that automatically verifies the general equilibrium, it is sufficient to impose this boundary condition at $z=L$, and the other condition at $z=-L$ will be satisfied automatically. Therefore, this condition is replaced by

$$
\int_{0}^{2 \pi} \int_{r_{i n f}}^{r_{s u p}} \sigma(r, L) \cdot e_{z} r \mathrm{~d} r \mathrm{~d} \theta=0
$$

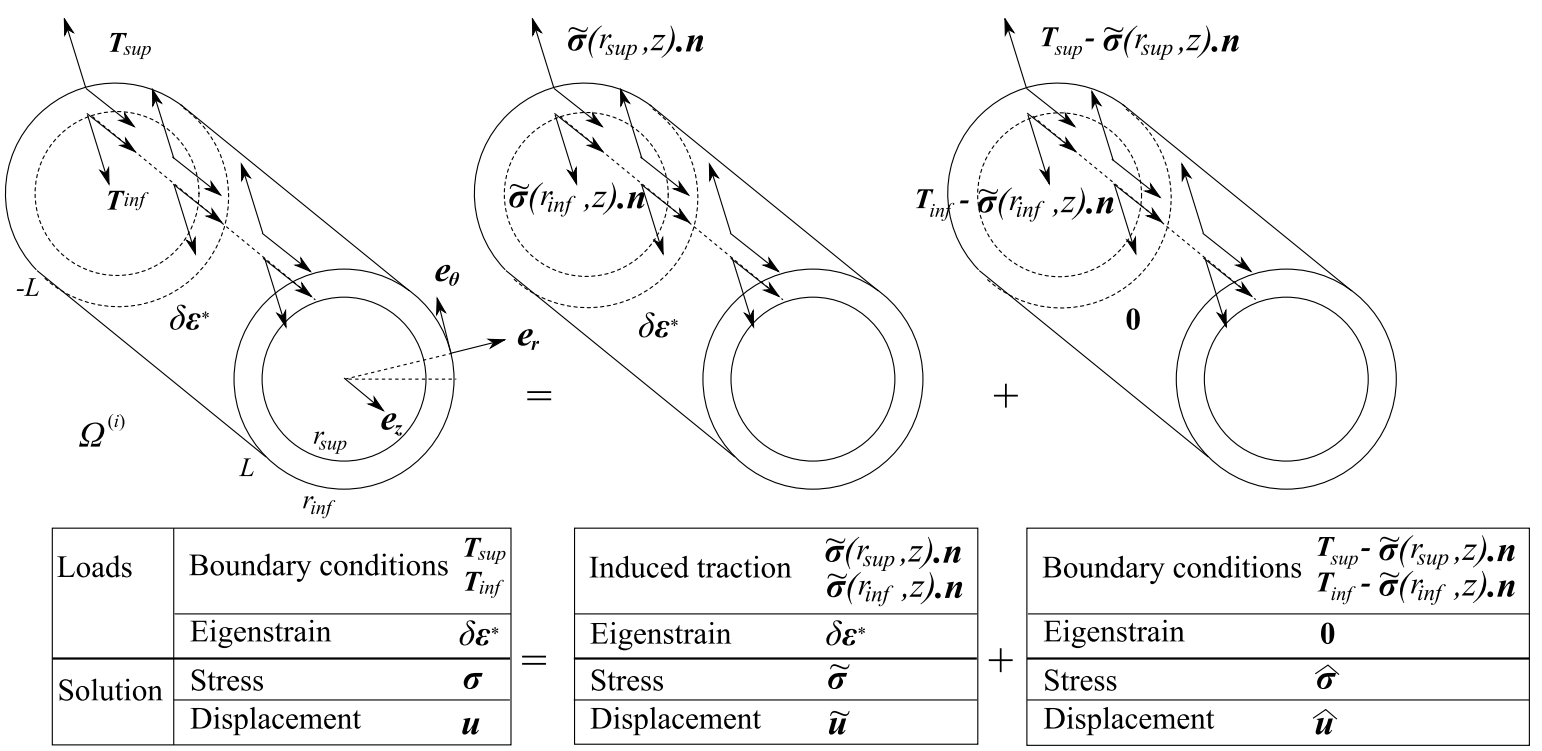

Figure 5: Decomposition in each layer

\section{Particular solution in each layer}

This section deals with the analytical determination of a particular solution $\tilde{\boldsymbol{u}}$ of the Navier equation (27), without specifying particular boundary conditions. One obtains after basic calculations the right-side term of (27):

$$
\operatorname{div}\left[\lambda \operatorname{tr}\left[\delta \boldsymbol{\varepsilon}^{*}\right] \boldsymbol{I}+2 \mu \delta \boldsymbol{\varepsilon}^{*}\right]=f_{r}^{*}(r, z) \boldsymbol{e}_{\boldsymbol{r}}+f_{z}^{*}(r, z) \boldsymbol{e}_{z}
$$

where

$$
\left\{\begin{array}{l}
f_{r}^{*}(r, z)=(\lambda+2 \mu) \frac{\partial \delta \varepsilon_{r r}^{*}}{\partial r}+\lambda\left(\frac{\partial \delta \varepsilon_{\theta \theta}^{*}}{\partial r}+\frac{\partial \delta \varepsilon_{z z}^{*}}{\partial r}\right)+2 \mu\left(\frac{\delta \varepsilon_{r r}^{*}}{r}-\frac{\delta \varepsilon_{\theta \theta}^{*}}{r}+\frac{\partial \delta \varepsilon_{r z}^{*}}{\partial z}\right) \\
f_{z}^{*}(r, z)=(\lambda+2 \mu) \frac{\partial \delta \varepsilon_{z z}^{*}}{\partial z}+\lambda\left(\frac{\partial \delta \varepsilon_{r r}^{*}}{\partial z}+\frac{\partial \delta \varepsilon_{\theta \theta}^{*}}{\partial z}\right)+2 \mu\left(\frac{\partial \delta \varepsilon_{r z}^{*}}{\partial r}+\frac{\delta \varepsilon_{r z}^{*}}{r}\right)
\end{array}\right.
$$

To give an analytical form to the right-side term (32), a function sequence, orthogonal with respect to the following scalar product, is needed:

$$
\langle f, g\rangle=\int_{r_{\text {inf }}}^{r_{\text {sup }}} r f(r) g(r) \mathrm{d} r
$$

where $f$ and $g$ are functions mapping $\left[r_{\text {inf }}, r_{\text {sup }}\right]$ onto $\mathbb{R}$. Consider the following function sequence:

$$
G_{m}: r \in\left[r_{\text {inf }}, r_{\text {sup }}\right] \mapsto J_{1}\left(x_{m} \frac{r}{r_{\text {sup }}}\right) Y_{1}\left(x_{m} \frac{r_{\text {inf }}}{r_{\text {sup }}}\right)-J_{1}\left(x_{m} \frac{r_{\text {inf }}}{r_{\text {sup }}}\right) Y_{1}\left(x_{m} \frac{r}{r_{\text {sup }}}\right)
$$

And consider $F_{m}$ is a primitive (up to a multiplicative constant) of $G_{m}$ :

$$
F_{m}: r \in\left[r_{\text {inf }}, r_{\text {sup }}\right] \mapsto J_{0}\left(x_{m} \frac{r}{r_{\text {sup }}}\right) Y_{1}\left(x_{m} \frac{r_{\text {inf }}}{r_{\text {sup }}}\right)-J_{1}\left(x_{m} \frac{r_{\text {inf }}}{r_{\text {sup }}}\right) Y_{0}\left(x_{m} \frac{r}{r_{\text {sup }}}\right)
$$


where $J_{\eta}$ and $Y_{\eta}$ are Bessel functions of order $\eta \in \mathbb{R}$ of the first and second kind, respectively. In addition, $x_{m}$ are successive positive roots of $x \mapsto J_{1}(x) Y_{1}\left(x \frac{r_{\text {inf }}}{r_{\text {sup }}}\right)-J_{1}\left(x \frac{r_{\text {inf }}}{r_{\text {sup }}}\right) Y_{1}(x)$. $G_{m}$ vanishes at $r=r_{\text {inf }}$ and $r=r_{\text {sup }}$. The following orthogonality property is proven in Appendix A:

$$
\left\langle G_{m}, G_{l}\right\rangle=\left\{\begin{array}{lll}
0 & \text { if } \quad m \neq l \\
M_{m}>0 & \text { if } \quad l=m
\end{array}\right.
$$

The proposed approach relies on Fourier series expansions of the right-side terms $f_{r}^{*}$ and $f_{z}^{*}$, according to the axial direction $z$ :

$$
\left\{\begin{array}{l}
f_{r}^{*}(r, z)=\sum_{k=-K}^{K} f_{k}^{r}(r) \exp \left(\frac{i k \pi}{L} z\right) \\
f_{z}^{*}(r, z)=\sum_{k=-K}^{K} f_{k}^{z}(r) \exp \left(\frac{i k \pi}{L} z\right)
\end{array}\right.
$$

where

$$
f_{k}^{r}(r)=\frac{1}{2 L} \int_{-L}^{L} f_{r}^{*}(r, z) \exp \left(\frac{-i k \pi}{L} z\right) \mathrm{d} z \quad \text { and } \quad f_{k}^{z}(r)=\frac{1}{2 L} \int_{-L}^{L} f_{z}^{*}(r, z) \exp \left(\frac{-i k \pi}{L} z\right) \mathrm{d} z
$$

The orthogonality property (36) is used to expand the functions $f_{k}^{r}(r)$ and $\partial f_{k}^{z}(r) / \partial r$ into a series based on the function sequence $G_{m}$. However, functions $G_{m}$ vanish at $r=r_{\text {inf }}$ and $r=r_{\text {sup }}$. Thus, the convergence can be difficult if $f_{k}^{r}(r)$ and $\partial f_{k}^{z}(r) / \partial r$ do not vanish at these points. It is therefore convenient to use the orthogonality property (36) not directly on $f_{k}^{r}(r)$ and $\partial f_{k}^{z}(r) / \partial r$ but on the following auxiliary functions, which vanish at $r=r_{\text {inf }}$ and $r=r_{\text {sup }}$ :

$$
\left\{\begin{array}{l}
g_{k}^{r}(r)=f_{k}^{r}(r)-\left(A_{k}^{r} J_{1}\left(\frac{r}{r_{\text {sup }}}\right)+B_{k}^{r} Y_{1}\left(\frac{r}{r_{\text {sup }}}\right)\right) \\
g_{k}^{z}(r)=f_{k}^{z}(r)-\left(A_{k}^{z} J_{0}\left(\frac{r}{r_{\text {sup }}}\right)+B_{k}^{z} Y_{0}\left(\frac{r}{r_{\text {sup }}}\right)\right)
\end{array}\right.
$$

where

$$
\left(\begin{array}{c}
A_{k}^{r} \\
B_{k}^{r}
\end{array}\right)=\left(\begin{array}{cc}
J_{1}\left(\frac{r_{\text {inf }}}{r_{\text {sup }}}\right) & Y_{1}\left(\frac{r_{\text {inf }}}{r_{\text {sup }}}\right) \\
J_{1}(1) & Y_{1}(1)
\end{array}\right)^{-1} \cdot\left(\begin{array}{c}
f_{k}^{r}\left(r_{\text {inf }}\right) \\
f_{k}^{r}\left(r_{\text {sup }}\right)
\end{array}\right)
$$

and

$$
\left(\begin{array}{c}
A_{k}^{z} \\
B_{k}^{z}
\end{array}\right)=-r_{\text {sup }}\left(\begin{array}{cc}
J_{1}\left(\frac{r_{\text {inf }}}{r_{\text {sup }}}\right) & Y_{1}\left(\frac{r_{\text {inf }}}{r_{\text {sup }}}\right) \\
J_{1}(1) & Y_{1}(1)
\end{array}\right)^{-1} \cdot\left(\begin{array}{c}
\frac{\partial f_{k}^{z}}{\partial f_{k}^{2}}\left(r_{\text {inf }}\right) \\
\frac{\partial f_{k}^{\prime}}{\partial r}\left(r_{\text {sup }}\right)
\end{array}\right)
$$

The following series expansion is based on an approach very similar to the classic Fourier-Bessel series expansion, detailed in [23]. The Fourier-Bessel series expansion is based on the function sequence $H_{m}: x \in[0, a] \mapsto J_{\eta}\left(y_{m}^{\eta} x\right)$, where $a>0$ and $\eta \in \mathbb{R}$ and where $y_{m}^{\eta}$ are the successive positive zeros of $x \mapsto J_{\eta}(a x)$. An orthogonality property similar to (36) also holds for the function sequence $H_{m}$. In addition, all continuous functions on $[0, a]$ can be written in the form $\sum_{m=1}^{+\infty} f_{m} H_{m}(x)$ (where $f_{m} \in \mathbb{R}$ ). Completeness is proven in [23] for an even more general function space. In this article, such a Fourier-Bessel series expansion is needed on the space of continuous functions on $\left[r_{i n f}, r_{\text {sup }}\right]$ instead of $[0, a]$. Thus, the only difference from the classic situation detailed in [23] is the starting point of the definition set, which is not zero. To overcome this difficulty, the Bessel function of the second kind was introduced in the function sequence $G_{m}$ (34). The proof of completeness developed in [23] could likely be adapted to the present situation, which is very similar. However, a simpler approach consists in projecting $g_{k}^{r}(r)$ and $\partial g_{k}^{z}(r) / \partial r$ onto the function space of finite dimension, generated by $\left(G_{m}\right)_{1 \leq m \leq M}$. Then using the orthogonality property (36) on the auxiliary functions (39), one obtains the projection

$$
\left\{\begin{array}{l}
f_{r}^{*}(r, z) \approx \sum_{k=-K}^{K}\left[\sum_{m=1}^{M} f_{m, k}^{r} G_{m}(r)+A_{k}^{r} J_{1}\left(\frac{r}{r_{\text {sup }}}\right)+B_{k}^{r} Y_{1}\left(\frac{r}{r_{\text {sup }}}\right)\right] \exp \left(\frac{i k \pi}{L} z\right) \\
f_{z}^{*}(r, z) \approx \sum_{k=-K}^{K}\left[\sum_{m=1}^{M} f_{m, k}^{z} F_{m}(r)+A_{k}^{z} J_{0}\left(\frac{r}{r_{\text {sup }}}\right)+B_{k}^{z} Y_{0}\left(\frac{r}{r_{\text {sup }}}\right)+C_{k}^{z}\right] \exp \left(\frac{i k \pi}{L} z\right)
\end{array}\right.
$$

where

$$
C_{k}^{z}=f_{k}^{z}\left(r_{\text {sup }}\right)-\sum_{m=1}^{M} f_{m, k}^{z} F_{m}\left(r_{\text {sup }}\right)-A_{k}^{z} J_{0}(1)-B_{k}^{z} Y_{0}(1)
$$


and where the coefficients $f_{m, k}^{r}$ and $f_{m, k}^{z}$ are evaluated numerically as follows:

$$
f_{m, k}^{r}=\frac{\left\langle g_{k}^{r}(r), G_{m}\right\rangle}{\left\langle G_{m}, G_{m}\right\rangle} \quad \text { and } \quad f_{m, k}^{z}=-\left(\frac{r_{s u p}}{x_{m}}\right) \frac{\left\langle\frac{\partial g_{k}^{z}(r)}{\partial r}, G_{m}\right\rangle}{\left\langle G_{m}, G_{m}\right\rangle}
$$

Furthermore, the inhomogeneous Navier equation (27) reads

$$
\left\{\begin{array}{l}
(\lambda+2 \mu)\left(\frac{\partial^{2} \widetilde{u}_{r}}{\partial r^{2}}+\frac{1}{r} \frac{\partial \widetilde{u}_{r}}{\partial r}-\frac{\widetilde{u}_{r}}{r^{2}}\right)+\mu \frac{\partial^{2} \widetilde{u}_{r}}{\partial z^{2}}+(\lambda+\mu) \frac{\partial^{2} \widetilde{u}_{z}}{\partial r \partial z}=f_{r}^{*}(r, z) \\
(\lambda+2 \mu) \frac{\partial^{2} \widetilde{u}_{z}}{\partial z^{2}}+\mu\left(\frac{\partial^{2} \widetilde{u}_{z}}{\partial r^{2}}+\frac{1}{r} \frac{\partial \widetilde{u}_{z}}{\partial r}\right)+(\lambda+\mu)\left(\frac{\partial^{2} \widetilde{u}_{r}}{\partial r \partial z}+\frac{1}{r} \frac{\partial \widetilde{u}_{r}}{\partial z}\right)=f_{z}^{*}(r, z)
\end{array}\right.
$$

A particular solution is sought as follows:

$$
\left\{\begin{array}{l}
\widetilde{u}_{r}(r, z)=\sum_{k=-K}^{K}\left[\sum_{m=1}^{M} u_{m, k}^{r} G_{m}(r)+U_{k}^{r} J_{1}\left(\frac{r}{r_{\text {sup }}}\right)+V_{k}^{r} Y_{1}\left(\frac{r}{r_{\text {sup }}}\right)\right] \exp \left(\frac{i k \pi}{L} z\right) \\
\widetilde{u}_{z}(r, z)=\sum_{k=-K}^{K}\left[\sum_{m=1}^{M} u_{m, k}^{z} F_{m}(r)+U_{k}^{z} J_{0}\left(\frac{r}{r_{\text {sup }}}\right)+V_{k}^{z} Y_{0}\left(\frac{r}{r_{\text {sup }}}\right)+W_{k}^{z}(r)\right] \exp \left(\frac{i k \pi}{L} z\right)
\end{array}\right.
$$

where

$$
W_{k}^{z}(r)= \begin{cases}\frac{C_{k}^{z} r^{2}}{4 \mu} & \text { if } \quad k=0 \\ -\frac{C_{k}^{z}}{(k \pi / L)^{2}(\lambda+2 \mu)} & \text { if } \quad k \neq 0\end{cases}
$$

By plugging (46) into (45) and identifying, one obtains

$$
\left(\begin{array}{c}
U_{k}^{r} \\
U_{k}^{z}
\end{array}\right)=\boldsymbol{S}_{k} \cdot\left(\begin{array}{c}
A_{k}^{r} \\
A_{k}^{z}
\end{array}\right) \text { and }\left(\begin{array}{c}
V_{k}^{r} \\
V_{k}^{z}
\end{array}\right)=\boldsymbol{S}_{k} \cdot\left(\begin{array}{c}
B_{k}^{r} \\
B_{k}^{z}
\end{array}\right) \text { and }\left(\begin{array}{c}
u_{m, k}^{r} \\
u_{m, k}^{z}
\end{array}\right)=\boldsymbol{S}_{m, k} \cdot\left(\begin{array}{c}
f_{m, k}^{r} \\
f_{m, k}^{z}
\end{array}\right)
$$

where

$$
\boldsymbol{S}_{k}=\left(\begin{array}{cc}
-(\lambda+2 \mu)\left(\frac{1}{r_{\text {sup }}}\right)^{2}-\mu\left(\frac{k \pi}{L}\right)^{2} & -(\lambda+\mu) \frac{1}{r_{\text {sut }}} \frac{i k \pi}{L} \\
(\lambda+\mu) \frac{1}{r_{\text {sup }}} \frac{i k \pi}{L} & -\mu\left(\frac{1}{r_{\text {sup }}}\right)^{2}-(\lambda+2 \mu)\left(\frac{k \pi}{L}\right)^{2}
\end{array}\right)^{-1}
$$

and

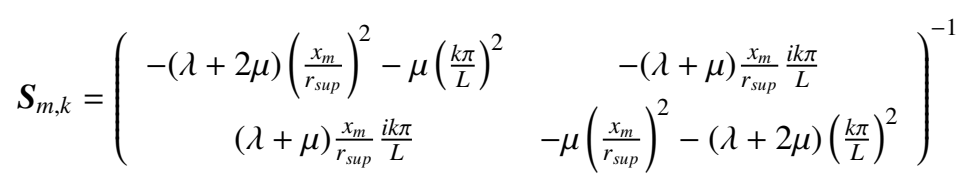

A particular displacement field $\widetilde{u}_{r}, \widetilde{u}_{z}$, the solution of the inhomogeneous Navier equation, has been established. Therefore, the associated stress field $\widetilde{\sigma}$ can be computed as well, with use of the behavior relation in (5). More precisely, a Fourier series expansion of $\widetilde{\sigma}$ is obtained since the displacement field is known as a Fourier series expansion, so component-wise

$$
\widetilde{\sigma}_{(. .)}(r, z)=\sum_{k=-K}^{K} \widetilde{\sigma}_{k}^{(.)}(r) \exp \left(\frac{i k \pi}{L} z\right)
$$

where the symbol (..) should be replaced by $r r, r z, z z$.

\section{Homogeneous solution}

As detailed in Section 4, the problem has been split into a particular solution of the inhomogeneous Navier equation (Section 5) and a homogeneous solution. This section is dedicated to the derivation of the homogeneous solution of the Navier equation (28) considering boundary conditions (29). Since the whole analytical solution 
derived in this article relies on Fourier series expansion along the axial direction, one should write additional contact pressure and shear stress as follows:

$$
\begin{aligned}
& P_{\text {inf }}(z)=\sum_{k=-K}^{K} P_{k}^{\text {inf }} \exp \left(i \frac{k \pi}{L} z\right) \mid S_{\text {inf }}(z)=\sum_{k=-K}^{K} S_{k}^{\text {inf }} \exp \left(i \frac{k \pi}{L} z\right) \\
& P_{\text {sup }}(z)=\sum_{k=-K}^{K} P_{k}^{\text {sup }} \exp \left(i \frac{k \pi}{L} z\right) \mid S_{\text {sup }}(z)=\sum_{k=-K}^{K} S_{k}^{s u p} \exp \left(i \frac{k \pi}{L} z\right)
\end{aligned}
$$

where

$$
\begin{array}{l|l}
P_{k}^{i n f}=\frac{1}{2 L} \int_{-L}^{L} P^{i n f}(z) \exp \left(-i \frac{k \pi}{L} z\right) \mathrm{d} z & S_{k}^{i n f}=\frac{1}{2 L} \int_{-L}^{L} S^{i n f}(z) \exp \left(-i \frac{k \pi}{L} z\right) \mathrm{d} z \\
P_{k}^{\text {sup }}=\frac{1}{2 L} \int_{-L}^{L} P^{\text {sup }}(z) \exp \left(-i \frac{k \pi}{L} z\right) \mathrm{d} z & S_{k}^{\text {sup }}=\frac{1}{2 L} \int_{-L}^{L} S^{\text {sup }}(z) \exp \left(-i \frac{k \pi}{L} z\right) \mathrm{d} z
\end{array}
$$

Boundary conditions (29) and (9) at $r=r_{\text {sup }}$ and $r=r_{\text {inf }}$ may be rewritten as

$$
\left\{\begin{array}{l}
\widehat{\boldsymbol{\sigma}}\left(r_{\text {sup }}, z\right) \cdot \boldsymbol{n}\left(r_{\text {sup }}, z\right)=\sum_{k=-K}^{K}\left[-\left(P_{k}^{\text {sup }}+\widetilde{\sigma}_{k}^{\text {rr }}\left(r_{\text {sup }}\right)\right) \boldsymbol{e}_{\boldsymbol{r}}+\left(S_{k}^{\text {sup }}-\widetilde{\sigma}_{k}^{r z}\left(r_{\text {sup }}\right)\right) \boldsymbol{e}_{z}\right] \exp \left(i \frac{k \pi}{L} z\right) \\
\widehat{\boldsymbol{\sigma}}\left(r_{\text {inf }}, z\right) \cdot \boldsymbol{n}\left(r_{\text {inf }}, z\right)=\sum_{k=-K}^{K}\left[\left(P_{k}^{\text {inf }}+\widetilde{\sigma}_{k}^{r r}\left(r_{\text {inf }}\right)\right) \boldsymbol{e}_{\boldsymbol{r}}+\left(S_{k}^{\text {inf }}+\widetilde{\sigma}_{k}^{r z}\left(r_{\text {inf }}\right)\right) \boldsymbol{e}_{z}\right] \exp \left(i \frac{k \pi}{L} z\right)
\end{array}\right.
$$

In the following, the homogeneous solution $\widehat{\boldsymbol{u}}, \widehat{\boldsymbol{\sigma}}$ verifying boundary conditions (54) and (30) is sought by use of biharmonic and harmonic potentials [17, 19,24]. To simplify the analytical procedure detailed in the following, the homogeneous solution $\widehat{\boldsymbol{u}}, \widehat{\boldsymbol{\sigma}}$ is decomposed into three contributions:

$$
\left\{\begin{array}{l}
\widehat{\boldsymbol{u}}=\widehat{\boldsymbol{u}}^{(a)}+\widehat{\boldsymbol{u}}^{(b)}+\widehat{\boldsymbol{u}}^{(c)} \\
\widehat{\boldsymbol{\sigma}}=\widehat{\boldsymbol{\sigma}}^{(a)}+\widehat{\boldsymbol{\sigma}}^{(b)}+\widehat{\boldsymbol{\sigma}}^{(c)}
\end{array}\right.
$$

The contribution with superscript (a) corresponds to purely biharmonic potentials and the contributions with superscripts $(b)$ and $(c)$ correspond to purely harmonic potentials.

\subsection{Biharmonic potentials}

A homogeneous solution $\widehat{\boldsymbol{u}}^{(a)}, \widehat{\boldsymbol{\sigma}}^{(a)}$ of the Navier equation (28) verifying boundary conditions (54) but not necessarily (30) is sought. A classical formulation using purely biharmonic potentials reads [17]

$$
\left\{\begin{array}{l}
2 \mu \widehat{u}_{r}^{(a)}=\frac{\partial^{2} \Phi}{\partial r \partial z} \\
2 \mu \widehat{u}_{z}^{(a)}=-\left[2(1-v)\left(\frac{\partial^{2} \Phi}{\partial r^{2}}+\frac{1}{r} \frac{\partial \Phi}{\partial r}\right)+(1-2 v) \frac{\partial^{2} \Phi}{\partial z^{2}}\right]
\end{array}\right.
$$

where $v=\lambda /(2(\lambda+\mu))$ is the Poisson ratio and where

$$
\Delta \Delta \Phi=0
$$

The biharmonic potential $\Phi$ is expanded into a Fourier series and modified Bessel functions as done by WeiszPatrault et al. [25]:

$$
\Phi(r, \theta, z)=\sum_{\substack{k=-K \\ k \neq 0}}^{K}\left(\frac{k \pi}{L}\right)^{-3}\left[\Phi_{k}\left(\frac{k \pi}{L} r\right) \frac{I_{0}^{\prime}\left(\frac{k \pi}{L} r\right)}{I_{0}^{\prime}\left(\frac{k \pi}{L} r_{i n f}\right)}+\varphi_{k} \frac{I_{0}\left(\frac{k \pi}{L} r\right)}{I_{0}\left(\frac{k \pi}{L} r_{\text {inf }}\right)}+\widehat{\Phi}_{k}\left(\frac{k \pi}{L} r\right) \frac{K_{0}^{\prime}\left(\frac{k \pi}{L} r\right)}{K_{0}^{\prime}\left(\frac{k \pi}{L} r_{\text {inf }}\right)}+\widehat{\varphi}_{k} \frac{K_{0}\left(\frac{k \pi}{L} r\right)}{K_{0}\left(\frac{k \pi}{L} r_{\text {inf }}\right)}\right] \exp \left(i \frac{k \pi}{L} z\right)
$$


where $I_{0}$ and $K_{0}$ are the zero-order modified Bessel functions of the first and second kind, respectively. Coefficients $\Phi_{k}, \varphi_{k}, \widehat{\Phi}_{k}$, and $\widehat{\varphi}_{k}$ are to be determined with the boundary conditions (54). The associated stress field is given by

$$
\left\{\begin{array}{l}
\widehat{\sigma}_{r r}^{(a)}=\left[-v \Delta \frac{\partial}{\partial z}+\frac{\partial^{3}}{\partial r^{2} \partial z}\right] \Phi \\
\widehat{\sigma}_{r z}^{(a)}=\left[-(1-v)\left(\frac{\partial^{3}}{\partial r^{3}}+\frac{1}{r} \frac{\partial^{2}}{\partial r^{2}}-\frac{1}{r^{2}} \frac{\partial}{\partial r}\right)+v \frac{\partial^{3}}{\partial r \partial z^{2}}\right] \Phi \\
\widehat{\sigma}_{\theta \theta}^{(a)}=\left[-v \Delta \frac{\partial}{\partial z}+\frac{1}{r} \frac{\partial^{2}}{\partial r \partial z}\right] \Phi \\
\widehat{\sigma}_{z z}^{(a)}=-\left[(2-v) \frac{\partial}{\partial z} \Delta-\frac{\partial^{3}}{\partial z^{3}}\right] \Phi
\end{array}\right.
$$

By inserting (58) into (59), one obtains

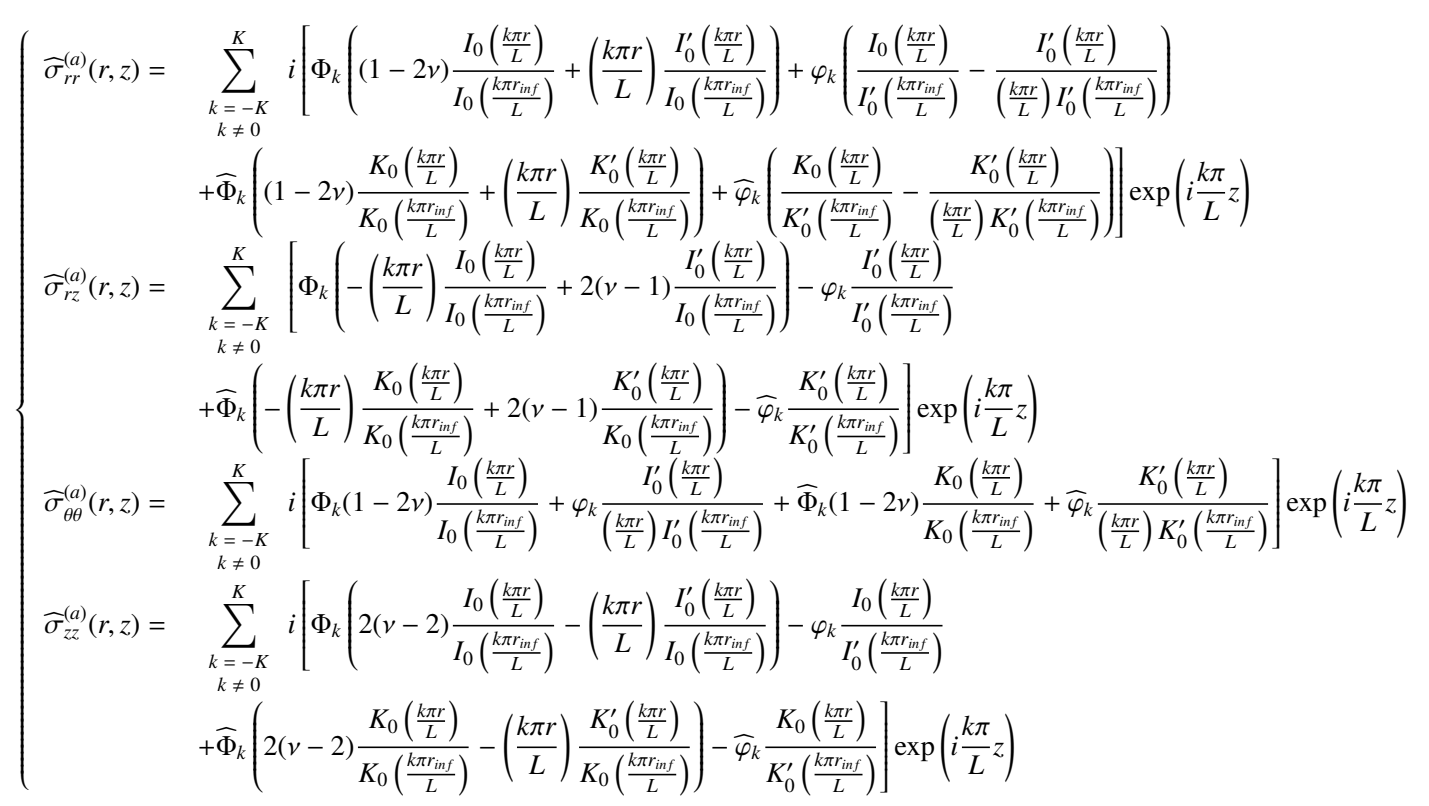

Using the boundary conditions (54) at $r=r_{\text {inf }}$ and $r=r_{\text {sup }}$, one obtains

$$
\left(\begin{array}{c}
\Phi_{k} \\
\varphi_{k} \\
\widehat{\Phi}_{k} \\
\widehat{\varphi}_{k}
\end{array}\right)=\boldsymbol{a}_{k}^{-1} \cdot\left(\begin{array}{c}
-\left(P_{k}^{i n f}+\widetilde{\sigma}_{k}^{r r}\left(r_{\text {inf }}\right)\right) \\
-\left(S_{k}^{i n f}+\widetilde{\sigma}_{k}^{r z}\left(r_{\text {inf }}\right)\right) \\
-\left(P_{k}^{(i+1)}+\widetilde{\sigma}_{k}^{r r}\left(r_{\text {sup }}\right)\right) \\
S_{k}^{(i+1)}-\widetilde{\sigma}_{k}^{r z}\left(r_{\text {sup }}\right)
\end{array}\right)
$$

where $\boldsymbol{a}_{k}$ is detailed coefficient-wise in Appendix B.

\subsection{Harmonic potentials}

Previous biharmonic potentials were expanded into a Fourier series (58), but $k=0$ was excluded from the expansion. Thus, the term independent of $z$ was not taken into account in the Fourier series expansion of the stress tensor $\widehat{\boldsymbol{\sigma}}^{(a)}(60)$. Therefore, boundary conditions (54) cannot be completely verified with the contribution $(a)$ only. In this section, a homogeneous solution $\widehat{\boldsymbol{u}}^{(b)}, \widehat{\boldsymbol{\sigma}}^{(b)}$ of the Navier equation (28) is sought such that $\widehat{\sigma}_{r r}^{(b)}$ and $\widehat{\sigma}_{r z}^{(b)}$ do not depend on $z$. Purely harmonic potentials are used to this end (as detailed in [17]):

$$
\left\{\begin{array}{l}
2 \mu \widehat{u}_{r}^{(b)}=\frac{\partial \phi}{\partial r}+z \frac{\partial \gamma}{\partial r} \\
2 \mu \widehat{u}_{z}^{(b)}=\frac{\partial \phi}{\partial z}+z \frac{\partial \gamma}{\partial z}-(3-4 v) \gamma
\end{array}\right.
$$


where

$$
\Delta \phi=\Delta \gamma=0
$$

The associated stress field is given by

$$
\left\{\begin{array}{l}
\widehat{\sigma}_{r r}^{(b)}=\frac{\partial^{2} \phi}{\partial r^{2}}+z \frac{\partial^{2} \gamma}{\partial r^{2}}-2 v \frac{\partial \gamma}{\partial z} \\
\widehat{\sigma}_{r z}^{(b)}=\frac{\partial^{2} \phi}{\partial r \partial z}+z \frac{\partial^{2} \gamma}{\partial r \partial z}-(1-2 v) \frac{\partial \gamma}{\partial r} \\
\widehat{\sigma}_{\theta \theta}^{(b)}=\frac{1}{r} \frac{\partial \phi}{\partial r}+\frac{z}{r} \frac{\partial \gamma}{\partial r}-2 v \frac{\partial \gamma}{\partial z} \\
\widehat{\sigma}_{z z}^{(b)}=\frac{\partial^{2} \phi}{\partial z^{2}}+z \frac{\partial^{2} \gamma}{\partial z^{2}}-2(1-v) \frac{\partial \gamma}{\partial z}
\end{array}\right.
$$

Harmonic polynomials according to $r$ and $z$ are sought for $\phi$ and $\gamma$ such that $\widehat{\sigma}_{r r}^{(b)}$ and $\widehat{\sigma}_{r z}^{(b)}$ do not depend on $z$. Thus,

$$
\left\{\begin{array}{l}
\phi=A_{0}\left(-\frac{r^{2}}{2}+z^{2}\right)+\frac{B_{0}}{r_{i n f}}\left(-\frac{3 r^{2} z}{2}+z^{3}\right)+C_{0}\left(r_{\text {inf }}\right)^{2} \ln \left(\frac{r}{r_{\text {inf }}}\right)+D_{0} r_{\text {inf }} z \ln \left(\frac{r}{r_{\text {inf }}}\right) \\
\gamma=-\frac{3}{1+4 v} \frac{B_{0}}{r_{\text {inf }}}\left(-\frac{r^{2}}{2}+z^{2}\right)-D_{0} r_{\text {inf }} \ln \left(\frac{r}{r_{\text {inf }}}\right)
\end{array}\right.
$$

Therefore, by plugging (65) into (62) and (64), one obtains

$$
\left\{\begin{array}{l}
2 \mu \widehat{u}_{r}^{(b)}=-A_{0} r-\frac{12 v B_{0}}{1+4 v} \frac{r z}{r_{\text {inf }}}+\frac{C_{0}\left(r_{\text {inf }}\right)^{2}}{r} \\
2 \mu \widehat{u}_{z}^{(b)}=2 A_{0} z+\frac{6}{1+4 v} \frac{B_{0}}{r_{\text {inf }}}\left(-r^{2}+z^{2}\right)+4(1-v) D_{0} r_{\text {inf }} \ln \left(\frac{r}{r_{\text {inf }}}\right)
\end{array}\right.
$$

and

$$
\left\{\begin{array}{l}
\widehat{\sigma}_{r r}^{(b)}=-A_{0}-C_{0}\left(\frac{r_{i n f}}{r}\right)^{2} \\
\widehat{\sigma}_{r z}^{(b)}=\frac{-6(1+v) B_{0}}{1+4 v} \frac{r}{r_{i n f}}+2(1-v) D_{0} \frac{r_{i n f}}{r} \\
\widehat{\sigma}_{\theta \theta}^{(b)}=-A_{0}+C_{0}\left(\frac{r_{i n f}}{r}\right)^{2} \\
\widehat{\sigma}_{z z}^{(b)}=2\left(A_{0}+\frac{6(1+v)}{1+4 v} \frac{B_{0} z}{r_{i n f}}\right)
\end{array}\right.
$$

Using the boundary conditions at $r=r_{\text {inf }}$ and $r=r_{\text {sup }}$, one obtains

$$
\left(\begin{array}{l}
A_{0} \\
C_{0}
\end{array}\right)=\left(\begin{array}{cc}
1 & 1 \\
1 & \left(\frac{r_{\text {inf }}}{r_{\text {sup }}}\right)^{2}
\end{array}\right)^{-1} \cdot\left(\begin{array}{c}
P_{0}+\sigma_{0}^{*, r r}\left(r_{\text {inf }}\right) \\
P_{0}^{(i+1)}+\sigma_{0}^{*, r r}\left(r_{\text {sup }}\right)
\end{array}\right)
$$

and

$$
\left(\begin{array}{c}
B_{0} \\
D_{0}
\end{array}\right)=\left(\begin{array}{cc}
\frac{-6(1+v)}{1+4 v} & 2(1-v) \\
\frac{-6(1+v)}{1+4 v} \frac{r_{\text {sup }}}{r_{\text {inf }}} & 2(1-v) \frac{r_{\text {inf }}}{r_{\text {sup }}}
\end{array}\right)^{-1} \cdot\left(\begin{array}{c}
-\left(S_{0}+\sigma_{0}^{*, r z}\left(r_{\text {inf }}\right)\right) \\
S_{0}^{(i+1)}-\sigma_{0}^{*, r z}\left(r_{\text {sup }}\right)
\end{array}\right)
$$

\subsection{Edge conditions}

The combination of contributions $(a)$ and $(b)$ satisfies boundary conditions (54) but not boundary conditions (30). In this section, a third (and final) solution $\widehat{\boldsymbol{u}}^{(c)}, \widehat{\boldsymbol{\sigma}}^{(c)}$ of the homogeneous solution of the Navier equation (28) is sought such that (30) is verified. Since the problem is axisymmetric, the boundary condition (30) reduces to

$$
\int_{r_{\text {inf }}}^{r_{\text {sup }}} \widehat{\sigma}_{z z}^{(c)}(r, L) r \mathrm{~d} r=-\int_{r_{\text {inf }}}^{r_{\text {sup }}}\left(\widetilde{\sigma}_{z z}+\widehat{\sigma}_{z z}^{(a)}+\widehat{\sigma}_{z z}^{(b)}\right)(r, L) r \mathrm{~d} r
$$

A formulation using purely harmonic potentials is used as in Section 6.2:

$$
\left\{\begin{array}{l}
\phi=\frac{E_{0} v}{1+v}\left(-\frac{r^{2}}{2}+z^{2}\right) \\
\gamma=-\frac{z E_{0}}{2(1+v)}
\end{array}\right.
$$


The only nonvanishing components of the associated displacements and stresses read

$$
\left\{\begin{array}{l}
2 \mu \widehat{u}_{r}^{(c)}=-\frac{E_{0} v}{1+v} r \\
2 \mu \widehat{u}_{z}^{(c)}=\frac{E_{0}}{1+v} z \\
\widehat{\sigma}_{z z}^{(c)}=E_{0}
\end{array}\right.
$$

Therefore, by use of (70), the last coefficient is obtained:

$$
E_{0}=-\frac{2}{\left(r_{\text {sup }}\right)^{2}-\left(r_{\text {inf }}\right)^{2}} \int_{r_{\text {inf }}}^{r_{\text {sup }}}\left(\widetilde{\sigma}_{z z}+\widehat{\sigma}_{z z}^{(a)}+\widehat{\sigma}_{z z}^{(b)}\right)(r, L) r \mathrm{~d} r
$$

\section{Numerical determination of contact stresses}

In previous sections, additional contact pressure $P_{\text {sup }}, P_{\text {inf }}$ and shear $S_{\text {sup }}, S_{\text {inf }}$ were arbitrary. In this section, the numerical determination of these contact stresses is detailed. It relies on the same principle as in the work of Weisz-Patrault et al. [10]. That is, contact stresses are evaluated by a minimization procedure using explicit contact laws defined in [10]. It consists in relating contact stresses to the position discontinuity between two successive layers. This discontinuity of nominal surfaces at each interface is constituted of the interpenetration $\llbracket r_{C} \rrbracket$ along the radial direction (associated with contact pressures) and the slide $\llbracket z_{C} \rrbracket$ along the axial direction (associated

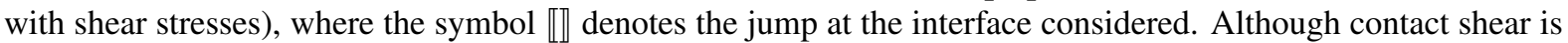
negligible in the numerical study detailed in this contribution, the determination of contact conditions is presented in general. Consider the following contact laws:

$$
P_{C}=f\left(\llbracket r_{C} \rrbracket\right) \text { and } S_{C}=g\left(\llbracket z_{C} \rrbracket\right)
$$

where $f$ and $g$ are simple functions defining the contact laws. In addition, $P_{C}$ and $S_{C}$ are the contact pressure and the shear stress at the interface considered. The coil consists of many layers, so contact pressure and shear at each interface are gathered in two vectors $\boldsymbol{P}$ and $\boldsymbol{S}$ as well as surface interpenetration $\llbracket \boldsymbol{r} \rrbracket$ and slides $\llbracket z \rrbracket$. Contact stresses are obtained by setting trial values $\widetilde{\boldsymbol{P}}$ and $\widetilde{\boldsymbol{S}}$ and computing the corresponding interpenetration $\llbracket \boldsymbol{r} \rrbracket$ and slides $\llbracket z \rrbracket$ with use of the mechanical model detailed in previous sections. Then the contact law (74) is used for each interface and gives another evaluation of contact stresses denoted by $\widetilde{\widetilde{\boldsymbol{P}}}$ and $\widetilde{\widetilde{\boldsymbol{S}}}$. Thus, contact stresses are determined by the solving of the following optimization problem:

$$
\boldsymbol{P}=\underset{\widetilde{\boldsymbol{P}} \geq 0}{\operatorname{argmin}}|\widetilde{\boldsymbol{P}}-\widetilde{\widetilde{P}}| \text { and } \boldsymbol{S}=\underset{\widetilde{\boldsymbol{S}}}{\operatorname{argmin}}|\widetilde{\boldsymbol{S}}-\widetilde{\widetilde{S}}|
$$

Contact stresses are $z$-dependent and should be evaluated for each time step, which leads to the solving of many optimization problems. Therefore, the analytical solution proposed for solving the mechanical problem enables us to obtain reasonable computation times.

\section{Validation of the analytical solution}

In this section, a numerical validation of the analytical solution developed in Sections 5 and 6 is proposed. Only one layer, subjected to an arbitrary eigenstrain, is modeled for this validation. The combination of several layers and the determination of contact stresses is not included in this section. An axisymmetric tube representing one layer of the coil was simulated by use of the finite element software program Cast3M developed by CEA [26]. The geometrical conditions, listed in Table 1, are chosen to be representative with respect to the application. Tractionfree boundary conditions are set at $r=r_{\text {inf }}$ and $r=r_{\text {sup }}$, and the only load consists in imposing an eigenstrain $\boldsymbol{\varepsilon}^{*}$ as listed in Table 2. These loading conditions necessitate very fine meshes to obtain reliable numerical results, which are presented in Figure 6 . The mesh density is defined by the number of elements along the radial direction $N_{r}=100$ and along the axial direction $N_{z}=1000$. The rather slow convergence of the finite element simulation toward the analytical solution, as a function of the mesh size, is illustrated in Figure 7 (where $N_{r}$ is fixed to 100). The comparison between the analytical solution and the finite element computation (at different axial positions) is presented in Figures 8 and 9. Good agreement is observed even though slight discrepancies are noticeable. These discrepancies are due to the lack of accuracy of the finite element computation. It can be observed that the 
boundary conditions are not verified perfectly: $\sigma_{r r}$ does not vanish at $r=r_{\text {inf }}$ and $r_{s u p}$, and $\sigma_{r z}$ does not vanish at $z=0$ (as it should because of the symmetry). In addition, the traction-free boundary condition at the edges $z= \pm L$ is not verified locally in the analytical solution. A weak condition is verified instead; that is, only the resultant forces vanish at the edges. The region affected by the local traction-free boundary condition spreads over a distance roughly equal to three times the thickness. Thus, this edge effect is negligible for applications where layers are very long compared with their thickness.

Table 1: Modeling parameters

\begin{tabular}{|l|ll|c|}
\hline External radius & $R_{\text {sup }}$ & $(\mathrm{mm})$ & 303 \\
\hline Internal radius & $R_{\text {inf }}$ & $(\mathrm{mm})$ & 300 \\
\hline Half length & $L$ & $(\mathrm{~mm})$ & $\begin{array}{c}50 \text { (condition 1) } \\
200 \text { (condition 2) }\end{array}$ \\
\hline
\end{tabular}

Table 2: Loading conditions

\begin{tabular}{|l|c|c|c|c|}
\hline & $\varepsilon_{r r}^{*}$ & $\varepsilon_{\theta \theta}^{*}$ & $\varepsilon_{z z}^{*}$ & $\varepsilon_{r z}^{*}$ \\
\hline Condition 1 & $P(r) \cos \left(\frac{\pi}{L} z\right)$ & $P(r) \cos \left(\frac{\pi}{L} z\right)$ & $P(r) \cos \left(\frac{\pi}{L} z\right)$ & 0 \\
\hline Condition 2 & 0 & 0 & 0 & $\sin \left(\frac{\pi}{L} z\right)$ \\
\hline
\end{tabular}

$P(r)=\left(r-R_{\text {sup }}\right)\left(r-R_{\text {inf }}\right) /\left(R_{\text {sup }} R_{\text {inf }}\right)$.

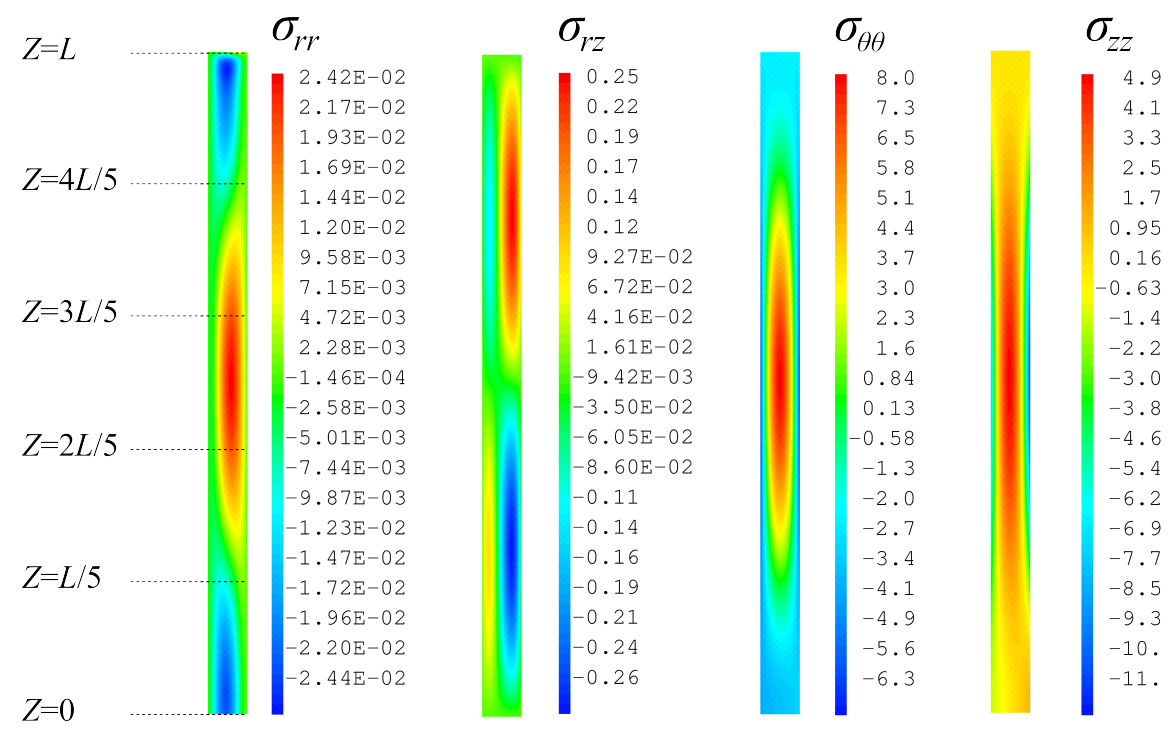

Figure 6: Finite element computation (condition 1) 

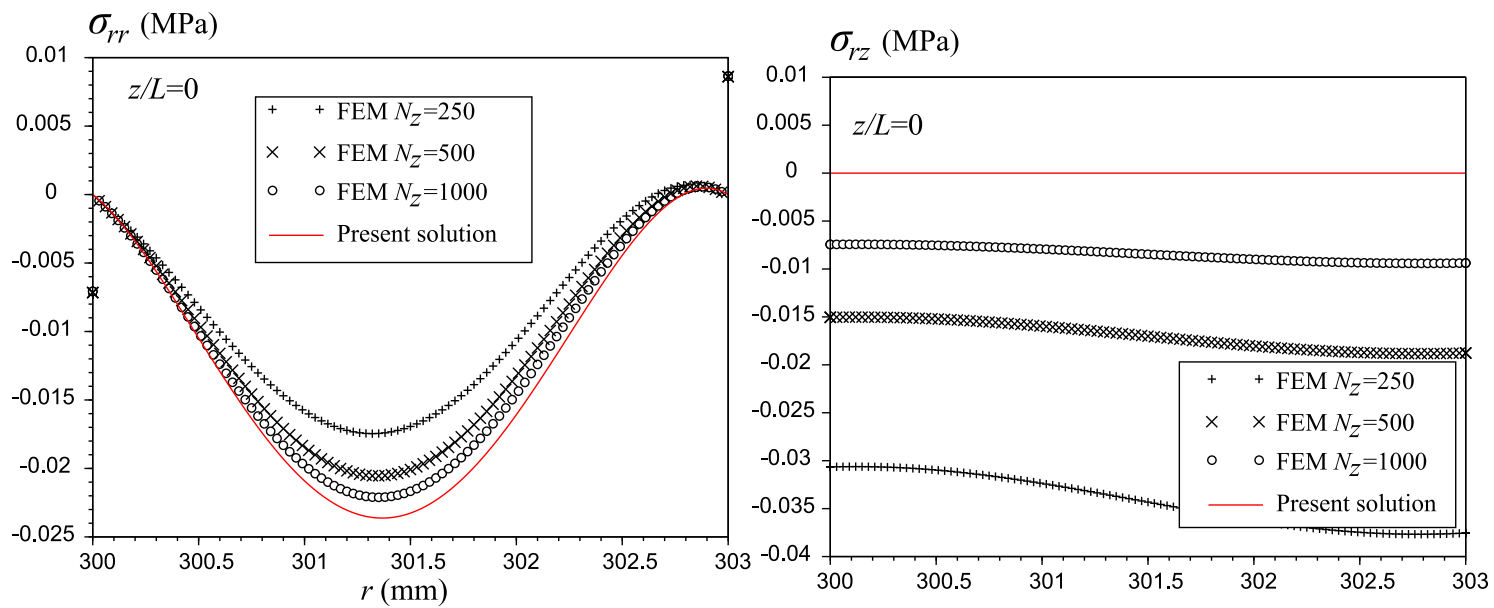

Figure 7: Convergence of the finite element computation (condition 1). FEM, finite element method.
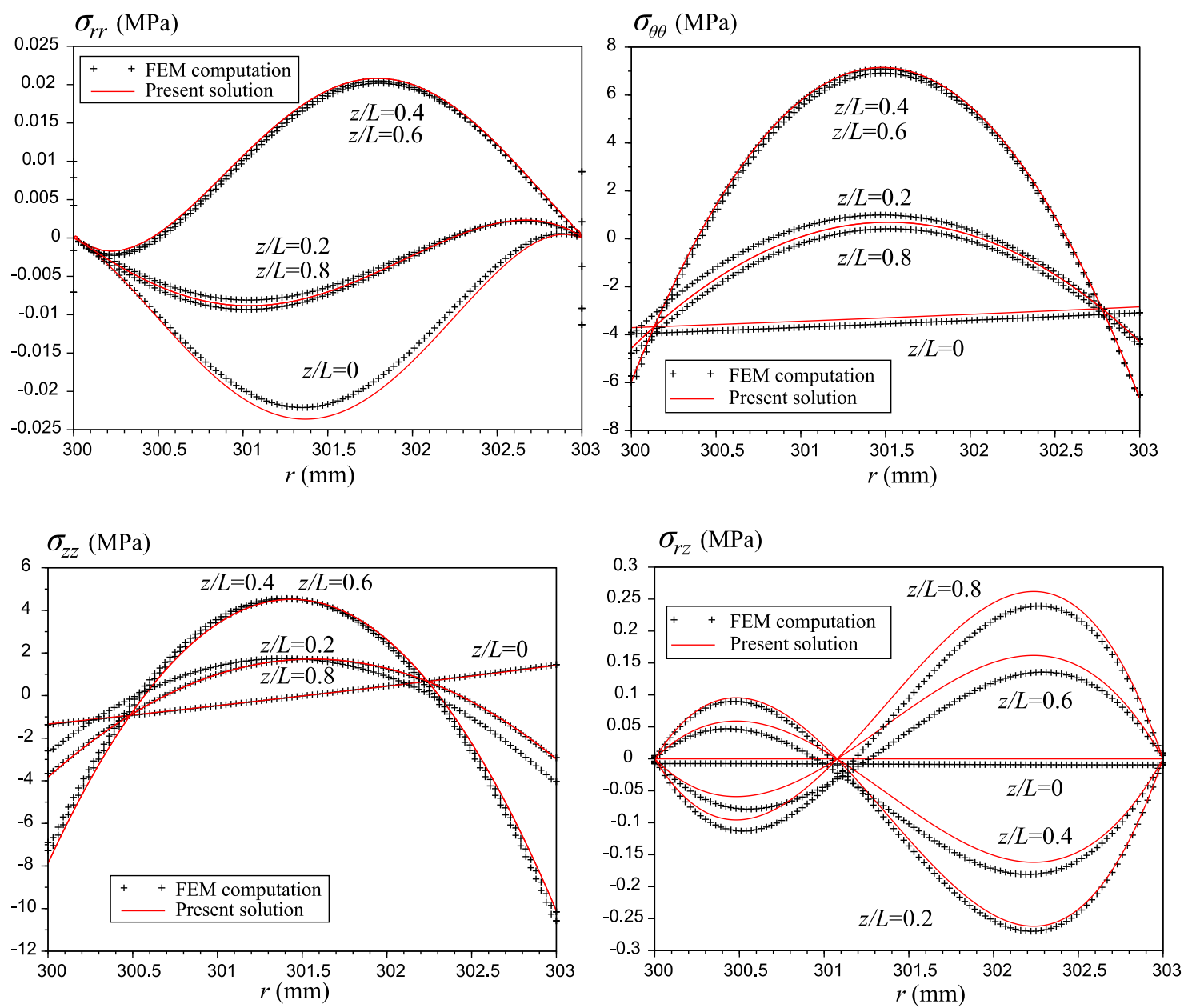

Figure 8: Validation by comparison with a finite element computation (condition 1). FEM, finite element method. 

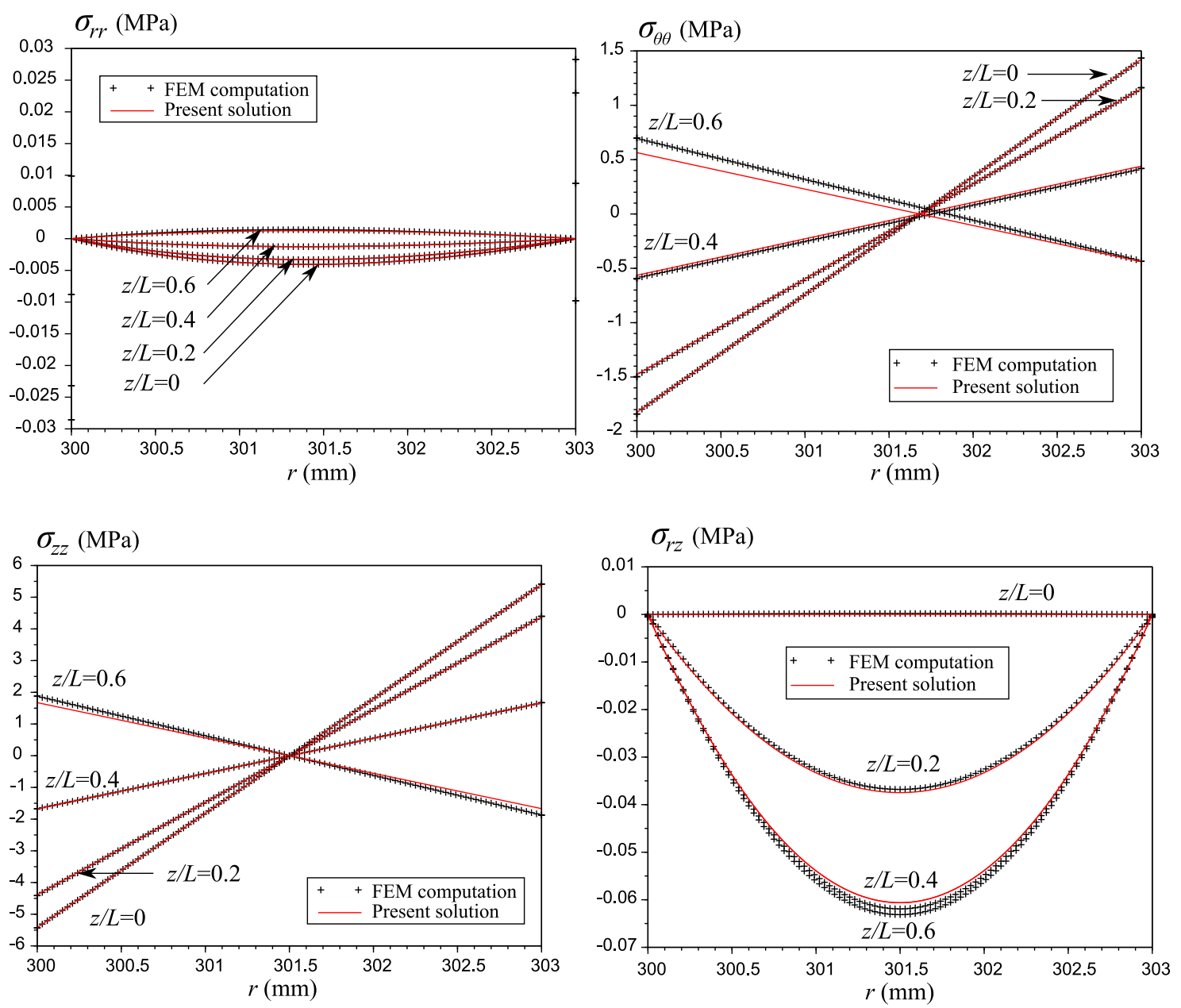

Figure 9: Validation by comparison with a Finite Element computation (condition 2). FEM, finite element method.

\section{Numerical results}

In this section, numerical results are obtained by use of models developed by Weisz-Patrault et al. [10] and Weisz-Patrault [13], on the one hand, and in the present contribution, on the other hand. The tested coiling parameters represent classic hot rolling conditions (e.g., for dual-phase steel grades). All parameters used for the simulation are listed in Table 3. Moreover, the Young's modulus as a function of temperature is extracted from [27]:

$$
E(T)=2.08 \times 10^{5}-1.90 \times 10^{2} T+1.19 T^{2}-2.82 \times 10^{-3} T^{3}+1.66 \times 10^{-6} T^{4}
$$

where $T$ is given in degrees Celsius. The yield stress of each phase as a function of temperature is needed. Grostabussiat-Petit [28] performed uniaxial tensile tests at different temperatures and identified the yield stress of austenite and bainite between $400{ }^{\circ} \mathrm{C}$ and $620^{\circ} \mathrm{C}$ and between $500{ }^{\circ} \mathrm{C}$ and $544{ }^{\circ} \mathrm{C}$, respectively. Other ferritic phases (ferrite and pearlite) are assumed to have the same yield stress as bainite in this example, even though proper yield stresses for all phases could have been used instead. The martensitic yield stress has been set to $800 \mathrm{MPa}$. Grostabussiat-Petit [28] proposed the following linear interpolation:

$$
\left\{\begin{array}{l}
\sigma_{0}^{Y}(T)=-0.147 \times T+183.14 \\
\sigma_{3}^{Y}(T)=-0.02 \times T+444.8
\end{array}\right.
$$

where $T$ is given in degrees Celsius.

The winding phase of the coiling process is simulated according to [10], and then the cooling phase is simulated according to [13]. The temperature field obtained is presented at different time steps in Figure 10. Considering the initial temperature, ferrite cannot be produced. Pearlite, bainite, and martensite phase proportion fields are given at different time steps in Figures 11, 12, and 13, respectively. 
When the coil is wound, the inner mandrel is removed to cool the coil more effectively. Contact pressures before and after mandrel removal are presented in Figure 14. It is clear that before removal of the mandrel, contact tends to concentrate more and more at the center when the number of layers increases. This is classically due to the geometrical profile of the incoming strip, which is assumed to be quadratic with respect to the axial direction. After removal of the mandrel, the contact pressure distribution is completely modified and tends to decrease significantly in amplitude and to concentrate at the center in the whole coil (and not only for the last layers). That is, removal of the internal support of the coil leads to loose contact, especially at the edges, where contact pressures were lower than at the center.

Thermal contact resistance is maximal when contact is lost; therefore, the coil cools more rapidly at the center, where a good contact is ensured, than at the edges, where the distance between two successive layers acts as a significant insulation. However, the first and last layers cool more rapidly at the edges because the air gaps tend to insulate them from the rest of the coil. In other words, the thermal capacity is much lower in these regions than at the center. The proportions of the different phases highly depend on temperature kinetics, and thus the global structures of the phase proportion fields are not surprisingly similar to the temperature variations.

The present contribution enables us to compute displacements and stresses when the coil cools down, considering, on the one hand, the initial state obtained at the end of the winding phase and after removal of the mandrel [10] and, on the other hand, all nonlinear contributions gathered as a total eigenstrain. When the coil cools down and undergoes phase transitions, contact pressures rapidly decrease, then localize and decrease again, as shown in Figure 15. These results depend on the chosen contact law that is relatively soft in this example. Instabilities are expected when contact pressures vanish all along an interface. In that situation adhesion between the corresponding layers is lost, and one should observe unwinding. It is clear that this issue is critical since contact pressure significantly decreases during cooling of the coil. It can be seen from Figure 15 that the proposed coiling conditions lead to this issue.

Another relevant result is the cumulative inelastic strain exhibited along the circumferential direction in Figure 16. Inelastic strain determines classic flatness defects obtained after uncoiling. For instance, one can use the simple purely elastic uncoiling model proposed by Weisz-Patrault et al. [10] to compute residual stresses on the basis of a flat reference. One of the most interesting ways to present the residual stress profile is in terms of residual curvature. In Figure 17, residual curvatures along the rolling direction denoted by $X$ and along the axial direction denoted by $Z$ are presented for different layers. Residual curvatures are presented before cooling (just after the elastic-plastic winding) and at the end of the cooling process to give insight into the extent to which thermal expansion, phase transitions, and transformation-induced plasticity contribute to the final residual stress. Significant residual stresses are produced during the coiling process and are dramatically increased and redistributed during the cooling phase, as shown in Figure 17. 
Table 3: Coiling conditions

\begin{tabular}{|l|cll|}
\hline External mandrel's radius & $R_{e x t}$ & 381 & $(\mathrm{~mm})$ \\
\hline Internal mandrel's radius & $R_{\text {int }}$ & 0 & $(\mathrm{~mm})$ \\
\hline Initial temperature & $T_{\text {ini }}$ & 750 & $(\mathrm{~K})$ \\
\hline External temperature & $T_{e x t}$ & 300 & $(\mathrm{~K})$ \\
\hline Heat transfer coefficient & $H$ & $0.4 \times 10^{-3}$ & $\left(\mathrm{~W} \mathrm{~mm}^{-2} \mathrm{~K}^{-1}\right)$ \\
\hline Applied nominal tension & $\sigma^{a}$ & 10 & $(\mathrm{MPa})$ \\
\hline Initial austenite proportion & $X_{1}^{\text {ini }}$ & 0.4 & \\
\hline Initial ferrite proportion & $X_{2}^{\text {in }}$ & 0.3 & \\
\hline Initial pearlite proportion & $X_{3}^{\text {ini }}$ & 0.3 & \\
\hline Initial bainite proportion & $X_{4}^{\text {ini }}$ & 0.0 & \\
\hline Initial martensite proportion & $X_{5}^{\text {ini }}$ & 0.0 & \\
\hline Half length & $L$ & 467 & $(\mathrm{~mm})$ \\
\hline Strip thickness at the center & $t_{c}$ & 3.3 & $(\mathrm{~mm})$ \\
\hline Strip thickness at the edges & $t_{e}$ & 3.261 & $(\mathrm{~mm})$ \\
\hline Number of cycles & $N_{\text {rev }}$ & 196 & \\
\hline Enthalpy variation for $p=2,3,4$ & $\Delta H_{p}$ & -0.4 & $\left.(\mathrm{~J} \mathrm{~mm})^{-3}\right)$ \\
\hline Enthalpy variation for $p=5$ & $\Delta H_{5}$ & -0.3 & $\left.(\mathrm{~J} \mathrm{~mm})^{-3}\right)$ \\
\hline Ferritic transition temperature & $A E_{3}$ & 1073 & $(\mathrm{~K})$ \\
\hline Pearlitic transition temperature & $A E_{1}$ & 823 & $(\mathrm{~K})$ \\
\hline Bainitic transition temperature & $B S$ & 713 & $(\mathrm{~K})$ \\
\hline Martensitic transition temperature & $M S$ & 693 & $(\mathrm{~K})$ \\
\hline Avrami exponents for all phase transitions & $n_{p}$ & 1.5 & \\
\hline Avrami coefficients for ferritic transformation & $k_{2}$ & 0.00008 & \\
\hline Avrami coefficients for pearlitic transformation & $k_{3}$ & 0.00015 & \\
\hline Avrami coefficients for bainitic transformation & $k_{4}$ & 0.00015 & \\
\hline Martensitic coefficient & $\alpha_{M S}$ & 0.045 & \\
\hline
\end{tabular}

Ferritic, pearlitic, and bainitic phase transitions are described by simple Avrami equations,

$$
\forall p \in\{2,3,4\}, X_{p}(t)=1-\exp \left(-k_{p} t^{n_{p}}\right),
$$

and the martensitic phase transition is described by

$$
X_{5}(t)=X_{r a}(t)\left[1-\exp \left(\alpha_{M S}(T(t)-M S)\right)\right]
$$

where $X_{r a}(t)$ is the retained austenite. 


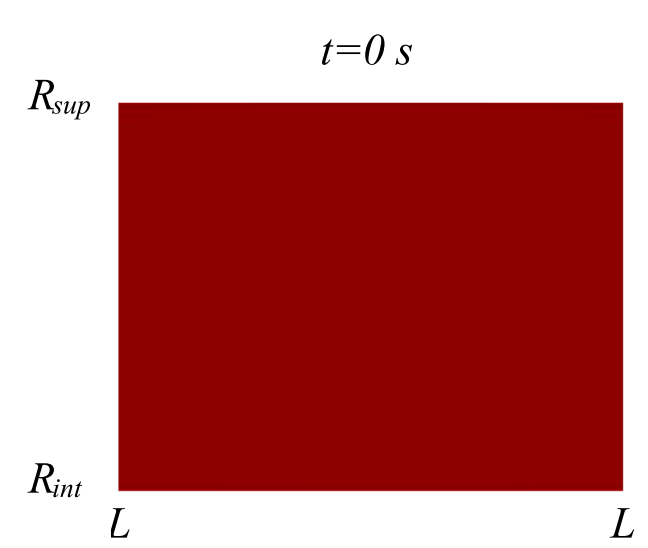

$t=380 \mathrm{~s}$

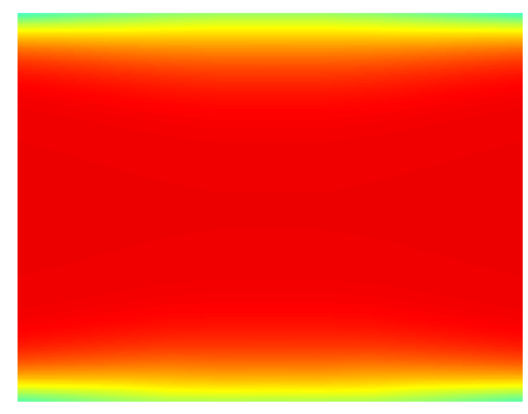

$t=1870 \mathrm{~s}$

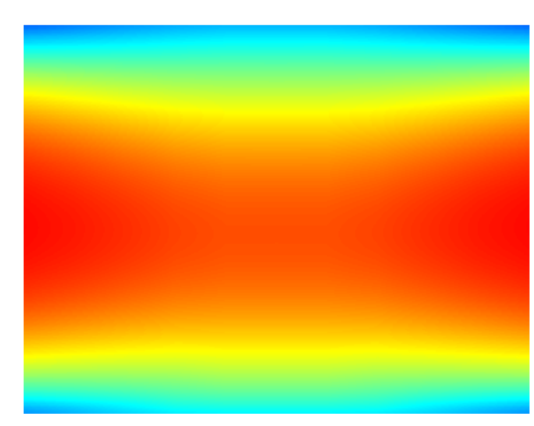

$t=8877 \mathrm{~s}$

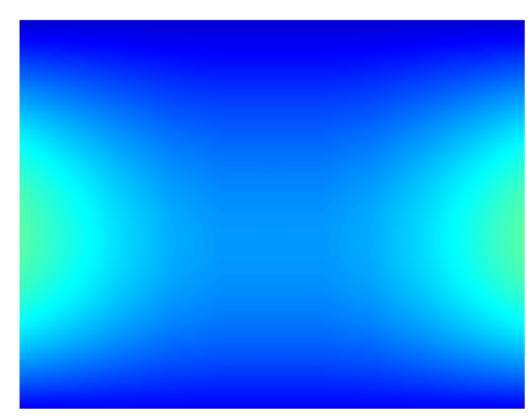

$t=160 \mathrm{~s}$

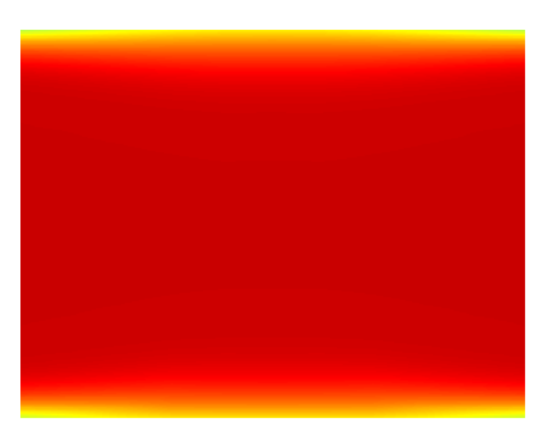

$t=850 \mathrm{~s}$

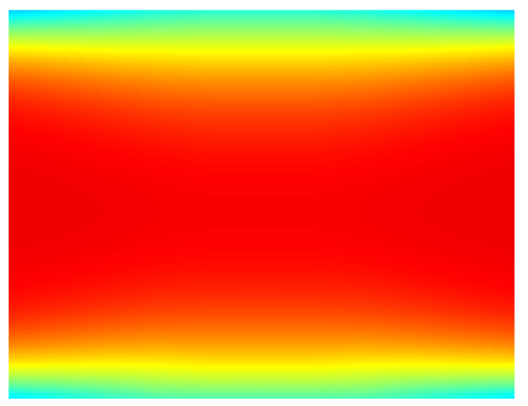

$t=4080 \mathrm{~s}$

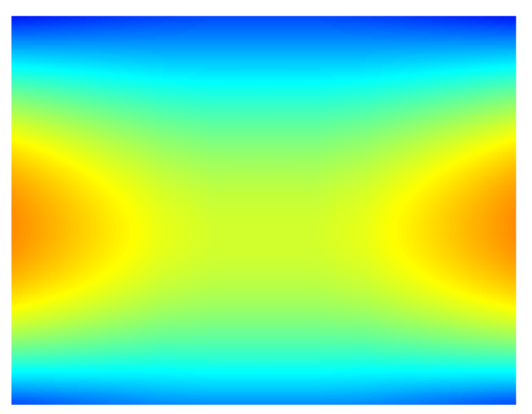

$t=18000 \mathrm{~s}$

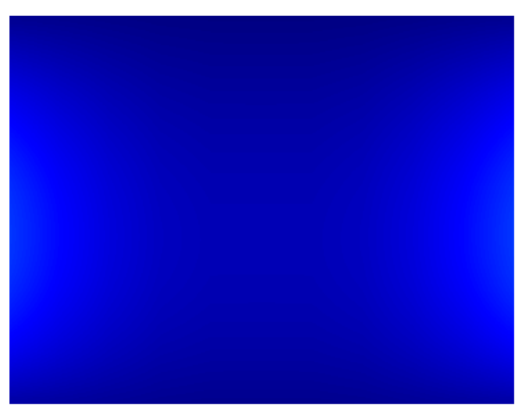

$T(\mathrm{~K})$

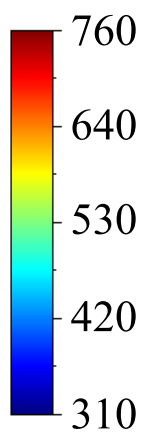

310

Figure 10: Temperature evolution 

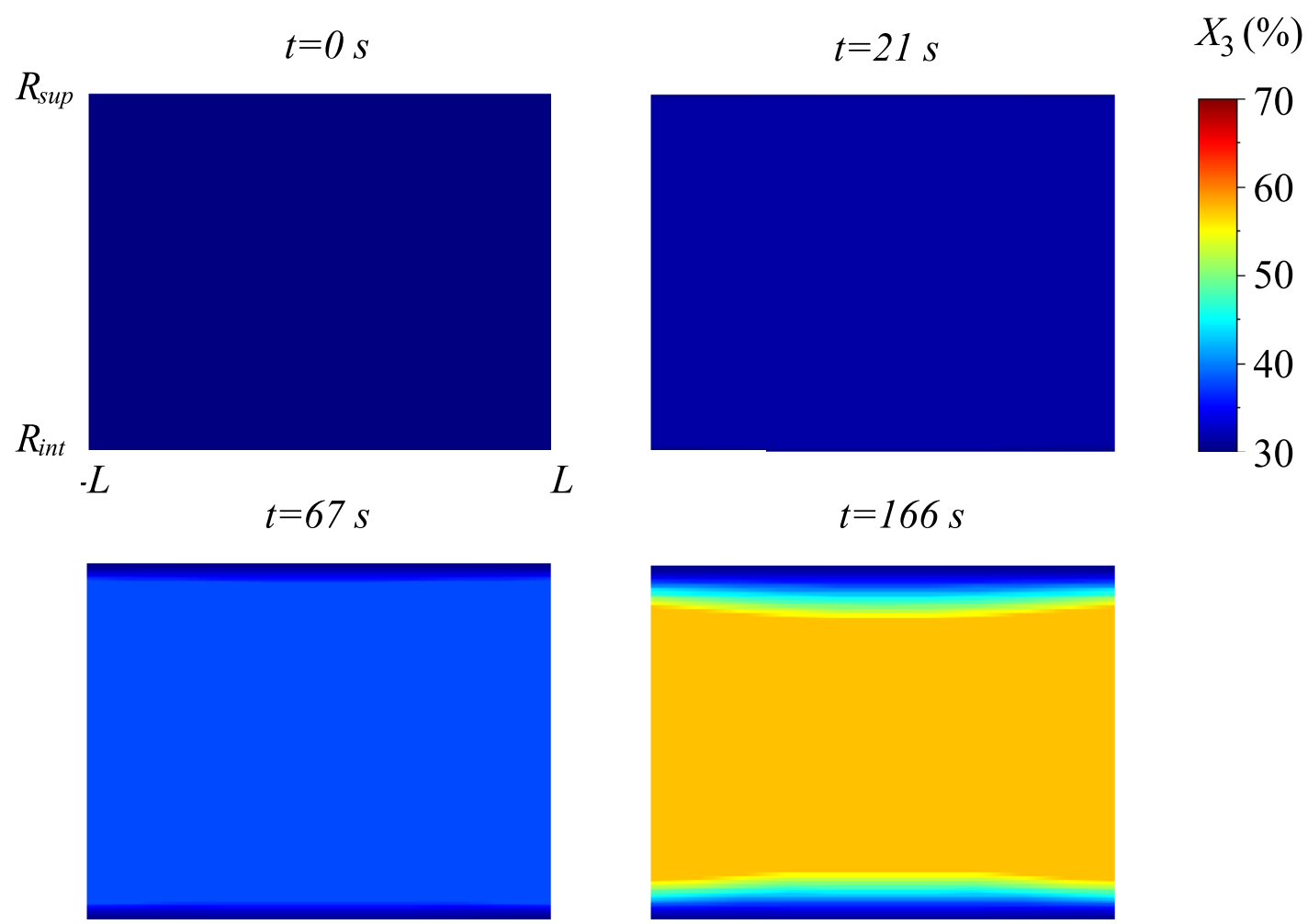

$t=380 \mathrm{~s}$

$t=18000 \mathrm{~s}$
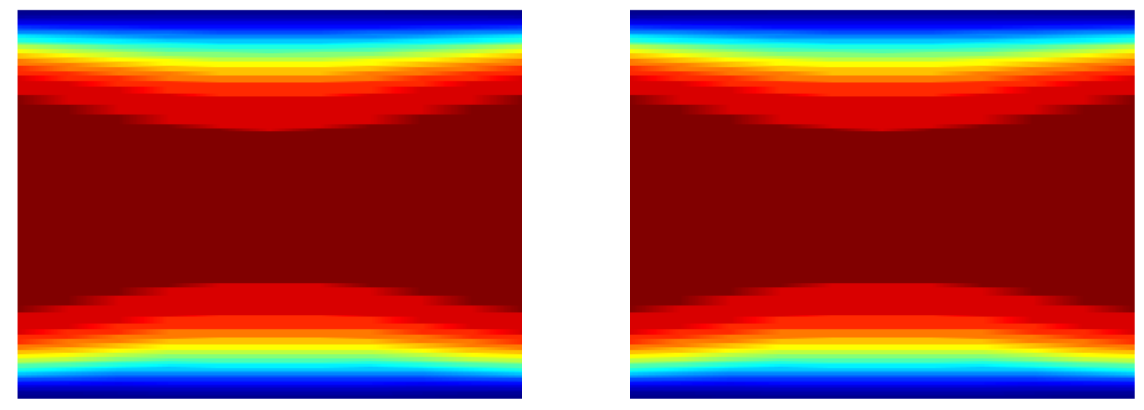

Figure 11: Pearlite proportion evolution 

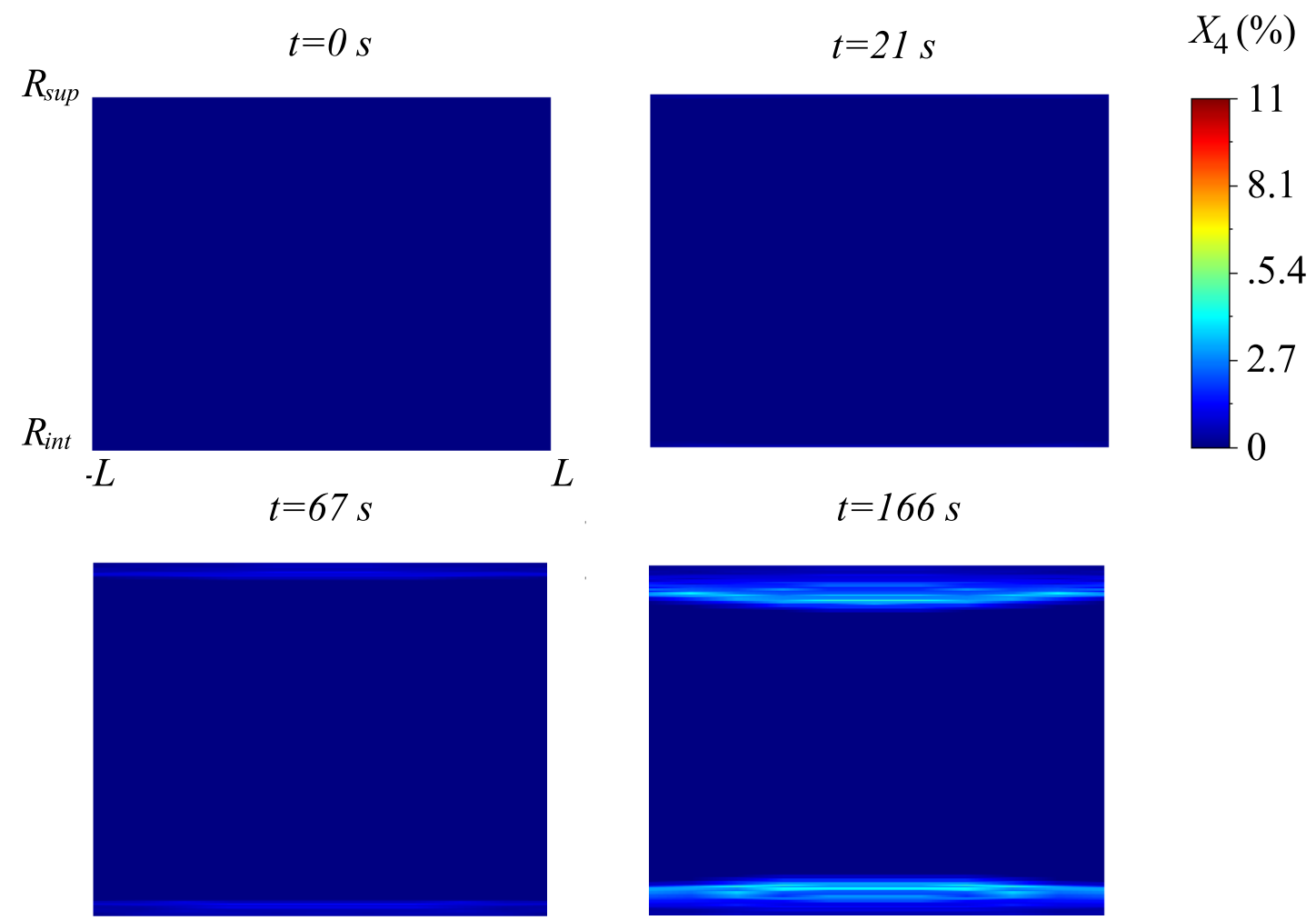

$t=380 \mathrm{~s}$

$t=18000 \mathrm{~s}$
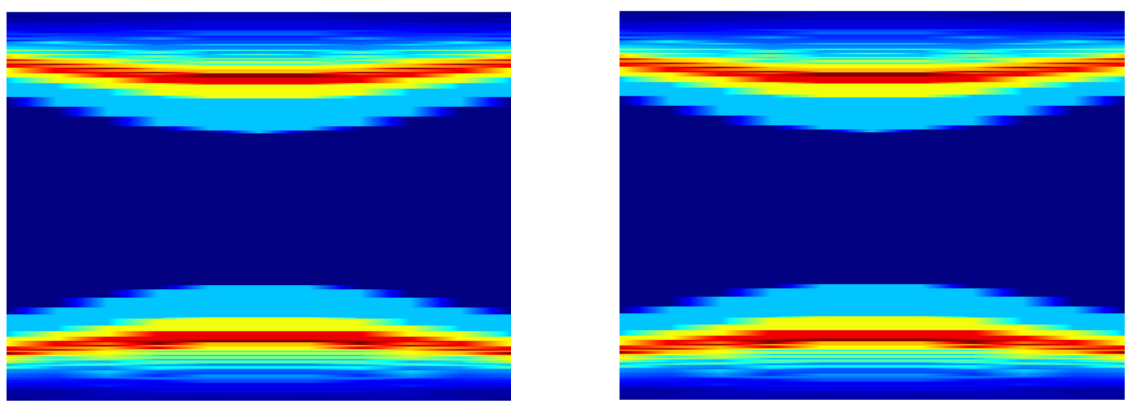

Figure 12: Bainite proportion evolution 

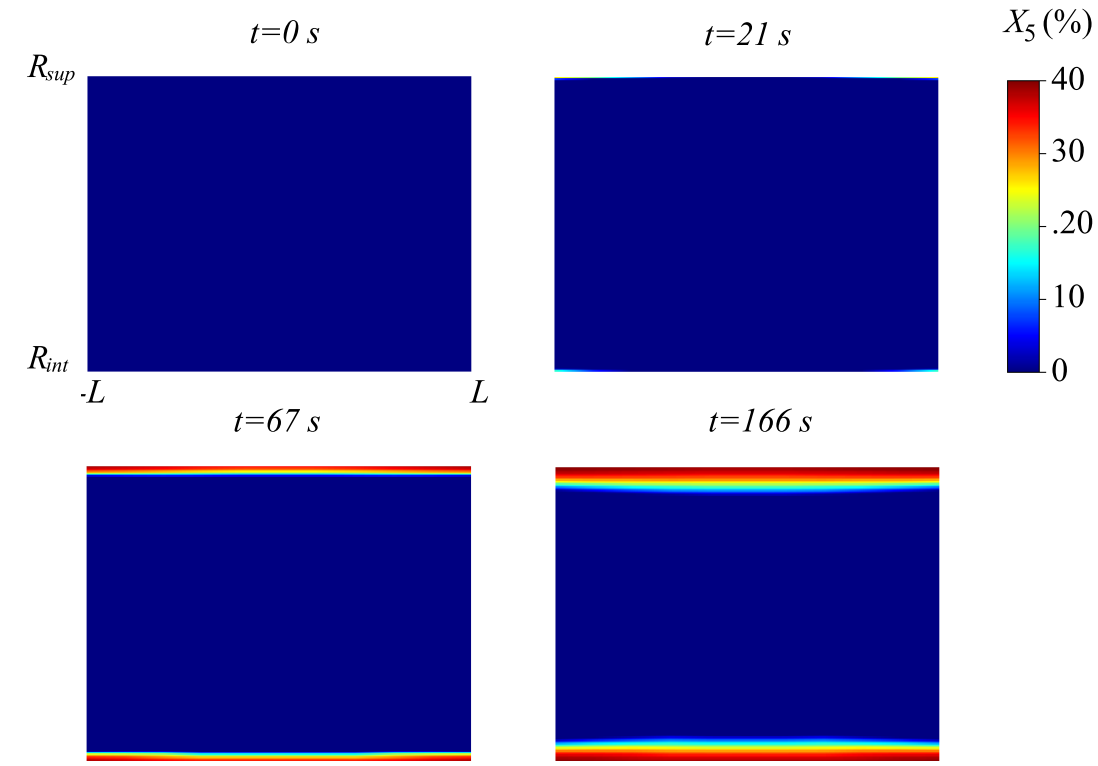

$t=380 \mathrm{~s}$

$t=18000 \mathrm{~s}$
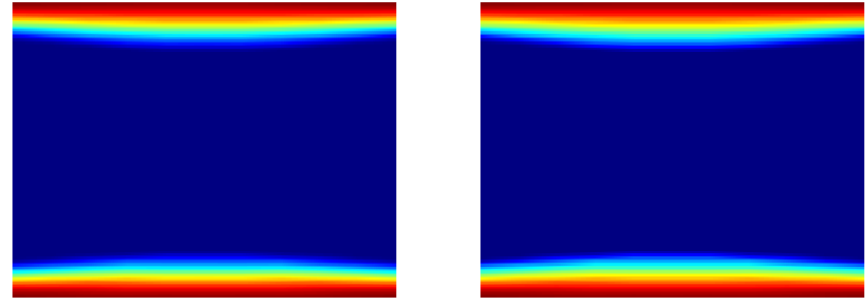

Figure 13: Martensite proportion evolution

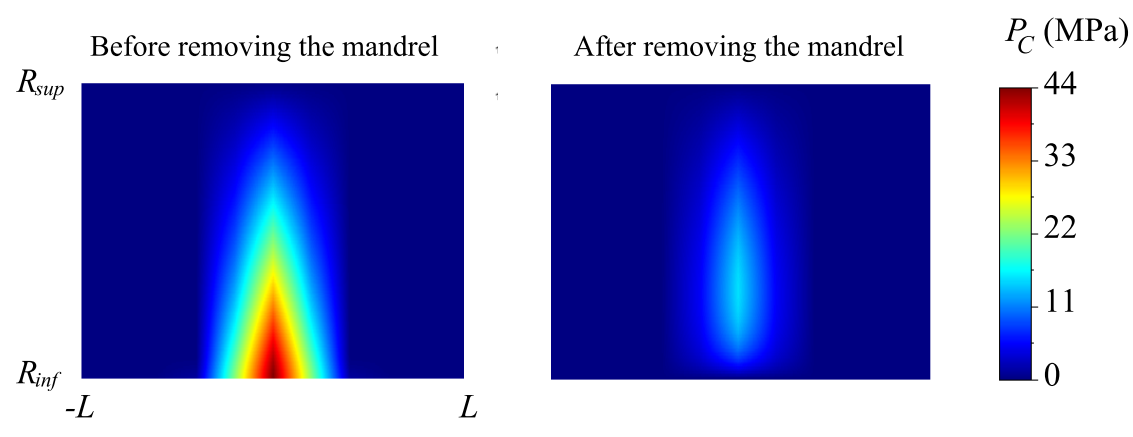

Figure 14: Contact pressure 

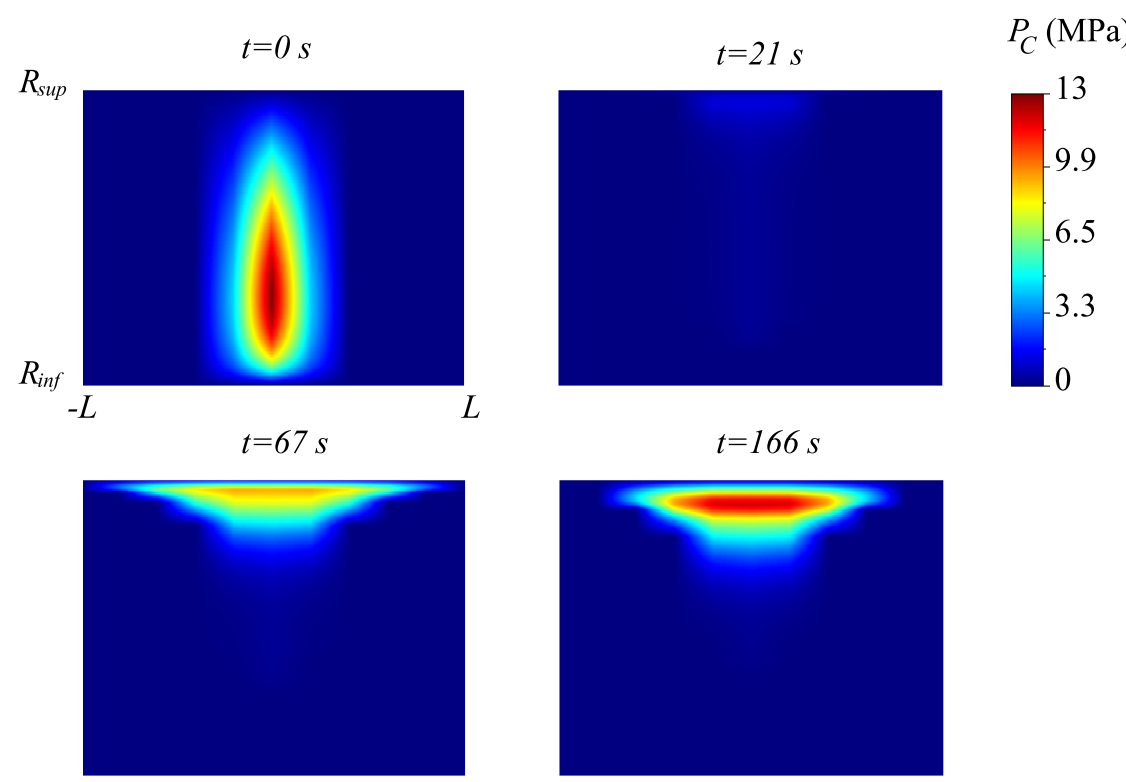

$t=380 \mathrm{~s}$

$$
t=850 \mathrm{~s}
$$
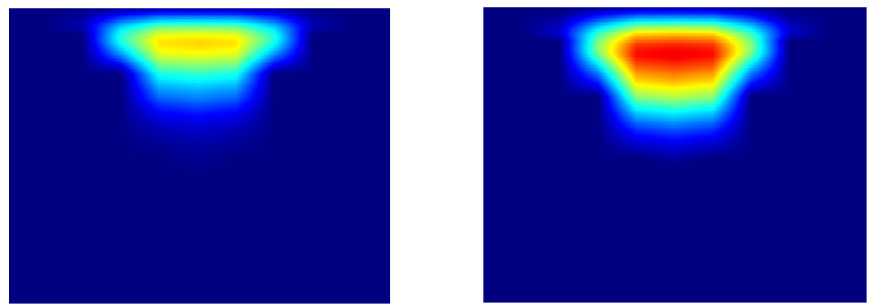

$t=1870 \mathrm{~s}$

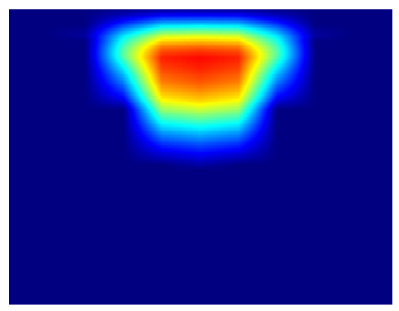

$t=4080 \mathrm{~s}$

$t=8877 \mathrm{~s}$

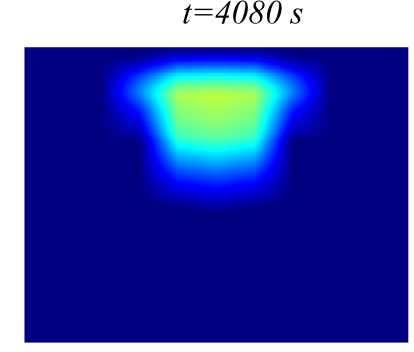

$$
t=18000 \mathrm{~s}
$$

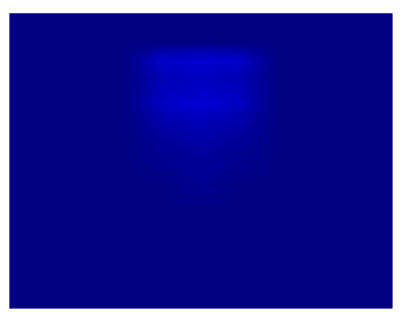

Figure 15: Contact pressure 

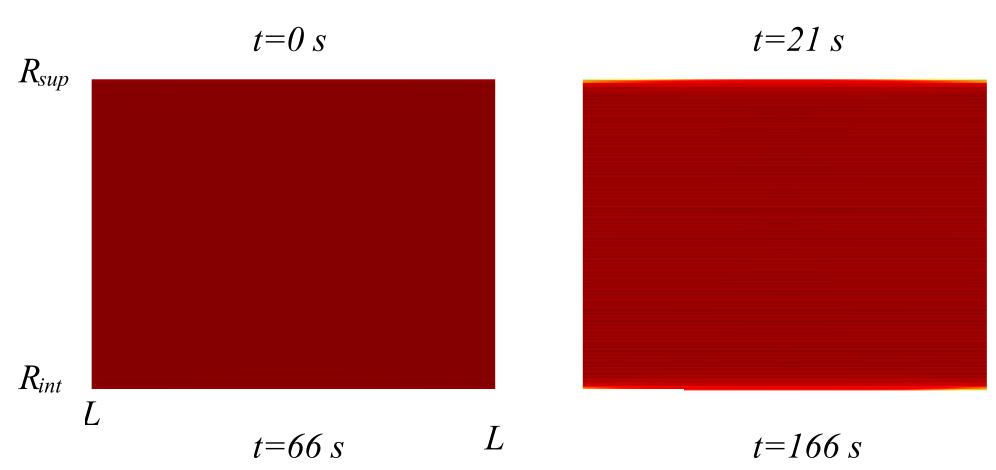

$\varepsilon_{\theta \theta}\left(10^{-3}\right)$
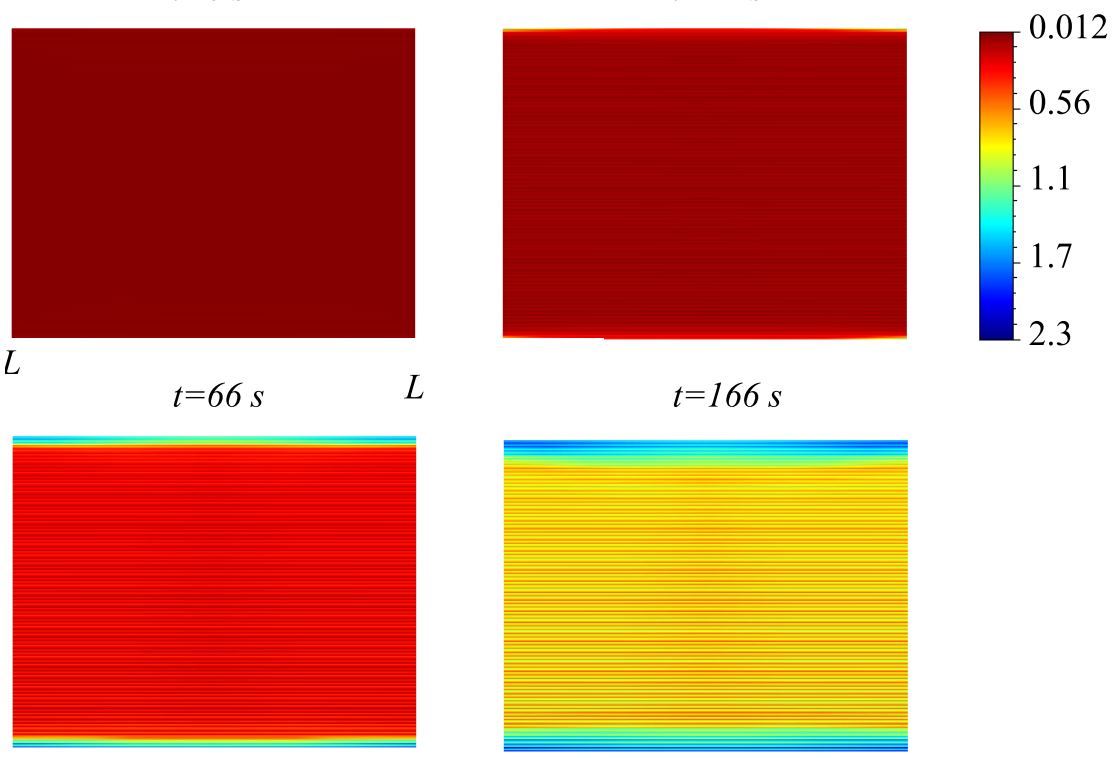

$t=380 \mathrm{~s}$

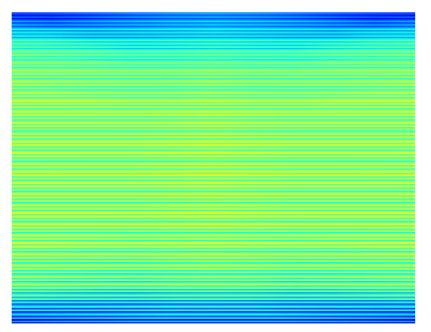

$$
t=850 \mathrm{~s}
$$

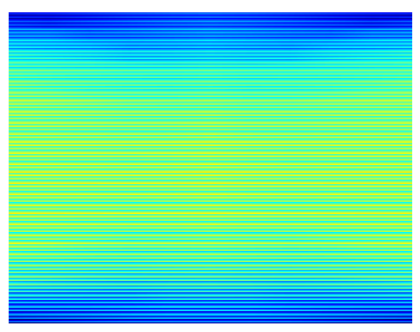

$t=1868 \mathrm{~s}$

$t=4078 \mathrm{~s}$
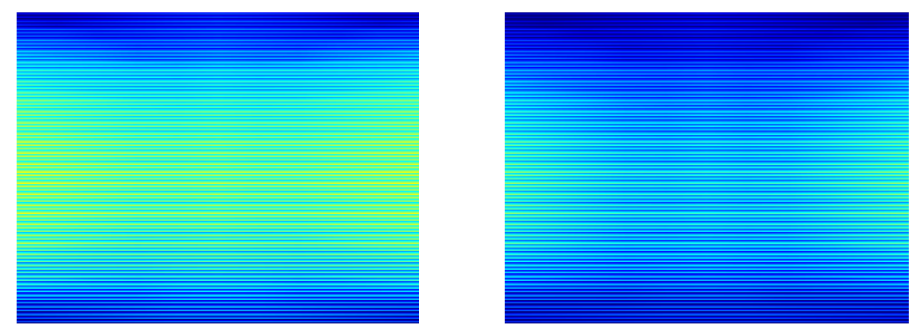

$t=8877 s$

$t=18000 \mathrm{~s}$
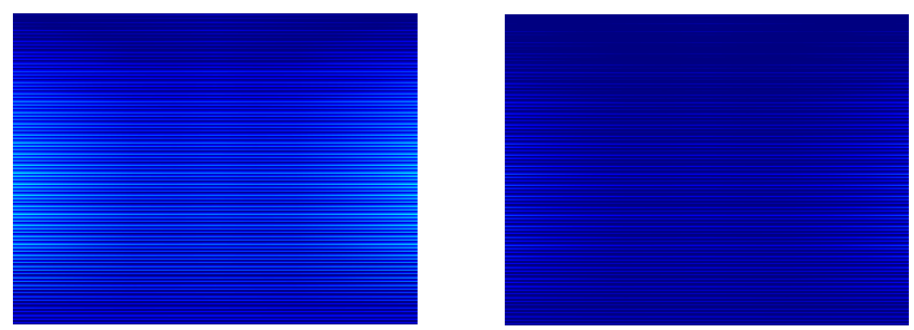

Figure 16: Irreversible eigenstrain ( $\theta \theta$ component) 


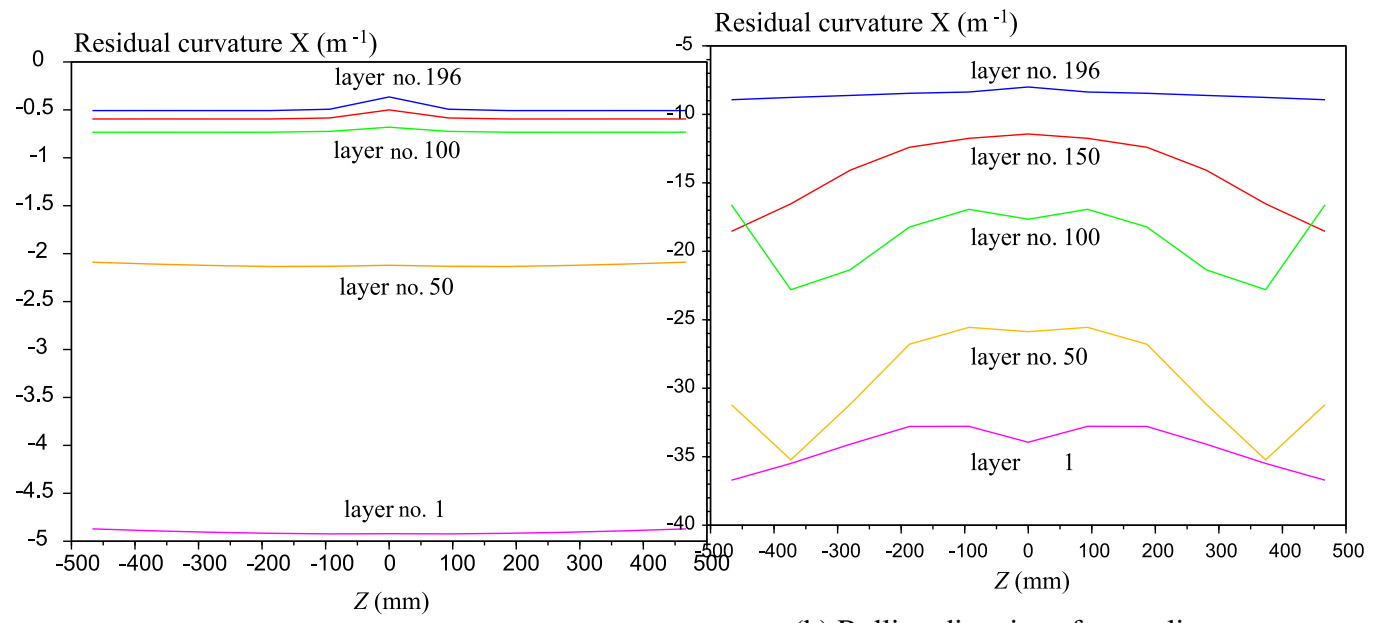

(a) Rolling direction before cooling

(b) Rolling direction after cooling

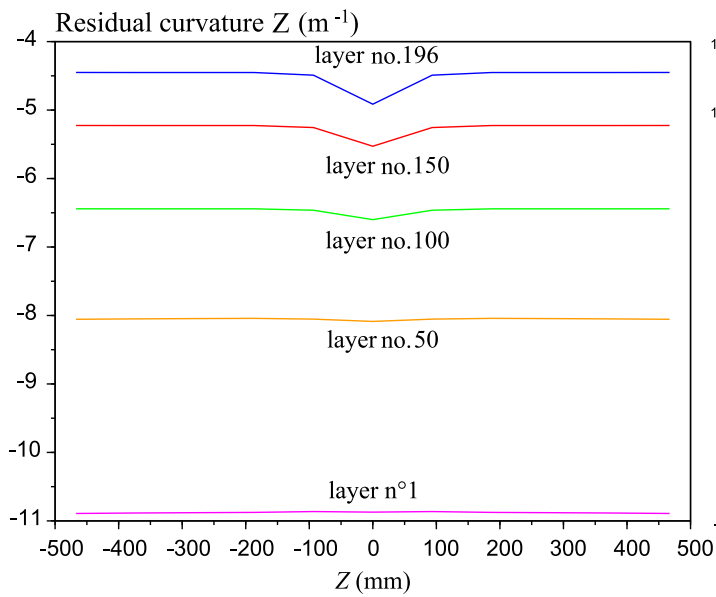

(c) Width direction before cooling

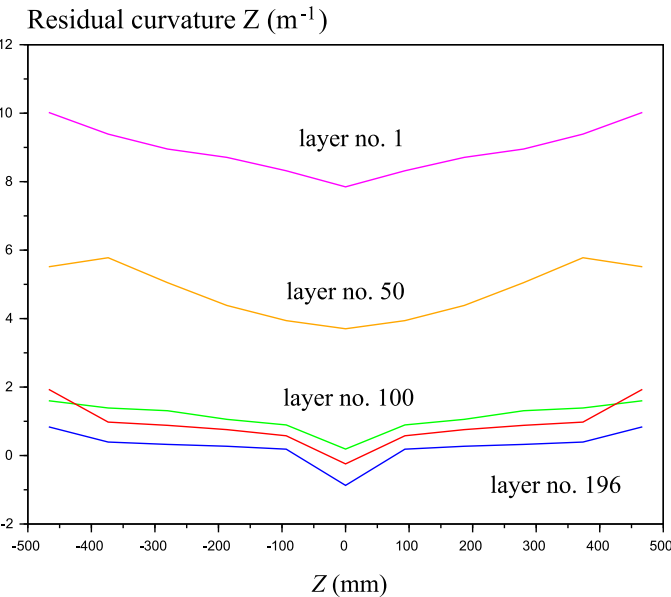

(d) Width direction after cooling

Figure 17: Residual curvature before and after cooling

\section{Conclusion}

A mixed analytical/numerical strategy has been developed to compute efficiently residual stresses induced by plastic deformation, phase transitions, and temperature variations in a coil. The analytical part of the proposed solution was verified by comparison with a finite element computation. The combination of previous models and the present solution enabled us to simulate the whole coiling process, including both winding and cooling of the coil. Parametric studies can be performed to optimize the process with respect to residual stress issues. In addition, this work also provides a tool for estimating inelastic strain during cooling, which is a significant input for computing coil sagging. Moreover, the example presented in this article shows that contact pressure tends to decrease when the coil cools down, which can lead to the unwinding of the coil if contact is lost. In addition, significant flatness defects are obtained after uncoiling.

\section{Acknowledgment}

The author gratefully acknowledge Nicolas Legrand (ArcelorMittal Global Research \& Development, East Chicago 3001 East Columbus Drive, East Chicago, IN 46312, USA) for fruitfully discussions. 


\section{Appendix A. Orthogonality and function basis}

The proof of orthogonality relies on several classic indefinite integrals (proven in [23]):

$$
\begin{aligned}
& \left\{\begin{array}{l}
\int r J_{1}(x r) J_{1}(y r) \mathrm{d} r=r \frac{y J_{1}(r x) J_{0}(r y)-x J_{0}(r x) J_{1}(r y)}{x^{2}-y^{2}} \\
\int r Y_{1}(x r) Y_{1}(y r) \mathrm{d} r=r \frac{y Y_{1}(r x) Y_{0}(r y)-x Y_{0}(r x) Y_{1}(r y)}{x^{2}-y^{2}} \\
\int r J_{1}(x r) Y_{1}(y r) \mathrm{d} r=r \frac{y J_{1}(r x) Y_{0}(r y)-x J_{0}(r x) Y_{1}(r y)}{x^{2}-y^{2}}
\end{array}\right. \\
& \left\{\begin{array}{l}
\int r J_{1}^{2}(x r) \mathrm{d} r=\frac{r^{2}}{2}\left(J_{1}^{2}(r x)-J_{0}(r x) J_{2}(r x)\right) \\
\int r Y_{1}^{2}(x r) \mathrm{d} r=\frac{r^{2}}{2}\left(Y_{1}^{2}(r x)-Y_{0}(r x) Y_{2}(r x)\right)
\end{array}\right.
\end{aligned}
$$

Hence

$$
\begin{aligned}
\int_{r_{\text {inf }}}^{r_{\text {sup }}} r G_{m}(r) G_{l}(r) \mathrm{d} r & =\int_{r_{\text {inf }}}^{r_{\text {sup }}} r\left(J_{1}\left(x_{m} \frac{r}{r_{\text {sup }}}\right) Y_{1}\left(x_{m} \frac{r_{\text {inf }}}{r_{\text {sup }}}\right)-J_{1}\left(x_{m} \frac{r_{\text {inf }}}{r_{\text {sup }}}\right) Y_{1}\left(x_{m} \frac{r}{r_{\text {sup }}}\right)\right) \\
& \times\left(J_{1}\left(x_{l} \frac{r}{r_{\text {sup }}}\right) Y_{1}\left(x_{l} \frac{r_{\text {inf }}}{r_{\text {sup }}}\right)-J_{1}\left(x_{l} \frac{r_{\text {inf }}}{r_{\text {sup }}}\right) Y_{1}\left(x_{l} \frac{r}{r_{\text {sup }}}\right)\right) \mathrm{d} r \\
& =\left\{\begin{array}{lll}
0 & \text { if } \quad m \neq l \\
M_{m}>0 & \text { if } \quad l=m
\end{array}\right.
\end{aligned}
$$




\section{Appendix B. Matrix coefficients}

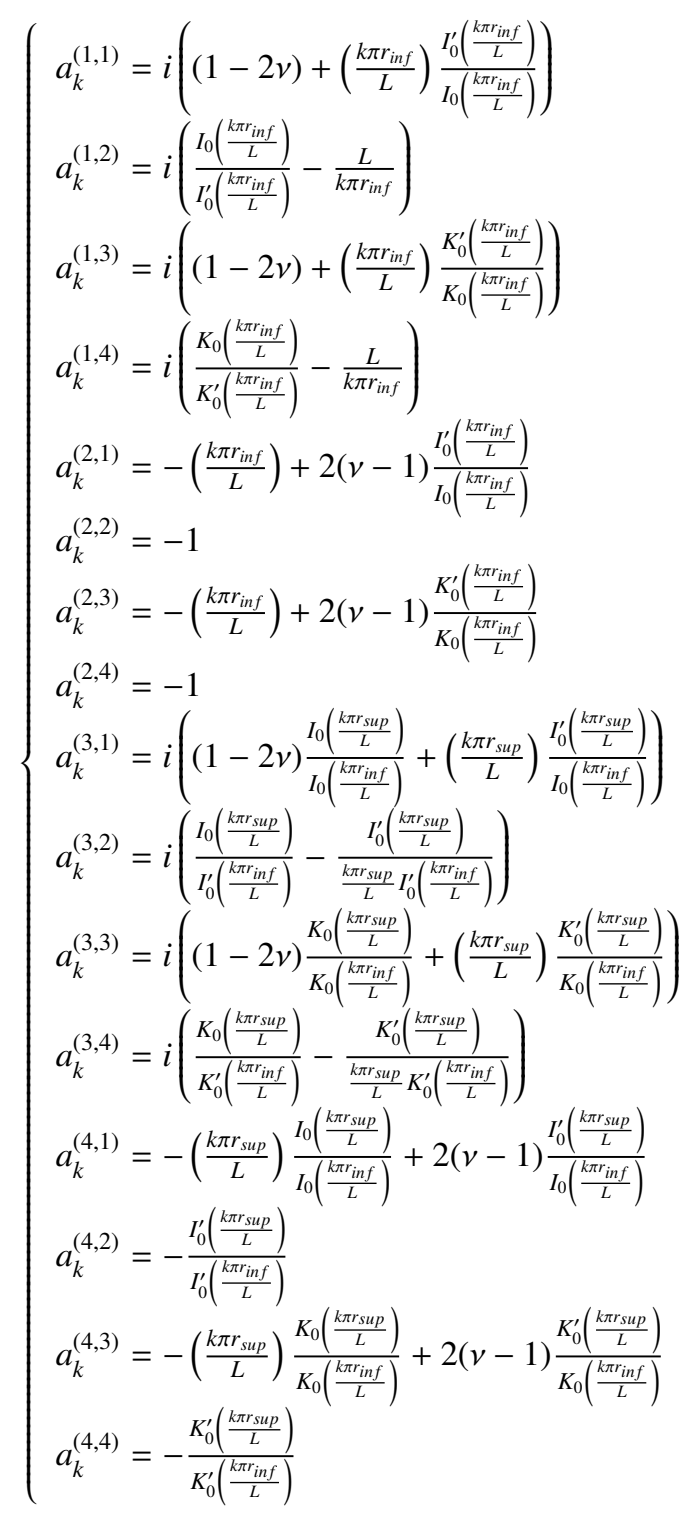

\section{References}

[1] S. Abdelkhalek, P. Montmitonnet, N. Legrand, P. Buessler, Coupled approach for flatness prediction in cold rolling of thin strip, International Journal of Mechanical Sciences 53 (2011) 661-675.

[2] W. Edwards, G. Boulton, The mystery of coil winding, in: 2001 Iron and Steel Exposition and AISE Annual Convention, pp. 2001-2017.

[3] W. Quach, J. Teng, K. Chung, Residual stresses in steel sheets due to coiling and uncoiling: a closed-form analytical solution, Engineering structures 26 (2004) 1249-1259.

[4] W. Quach, J. Teng, K. Chung, Residual stresses in press-braked stainless steel sections, ii: Press-braking operations, Journal of Constructional Steel Research 65 (2009) 1816-1826.

[5] C. D. Moen, T. Igusa, B. W. Schafer, Prediction of residual stresses and strains in cold-formed steel members, Thin-walled structures 46 (2008) 1274-1289.

[6] J. Hudzia, F. Ferrauto, P. Gevers, Stress calculation applied to a coil, and optimization of coiling tension, Cah. Inf. Tech. Rev. Metall. 91 (1994) 937-943. 
[7] D. Kedl, using a two dimensional winding model to predict wound roll stresses that occur due to circumferential steps in core diameter or to cross-web caliper variation, in: Proceedings of the Second International Conference on Web Handling, pp. 99-112.

[8] J. L. Hinton, A Study on the Effects of Coil Wedge During Rewinding of Thin Gauge Metals, Ph.D. thesis, Wright State University, 2011.

[9] J. L. Hinton, A. S. Malik, R. V. Grandhi, An airy function to rapidly predict stresses in wound metal strip having asymmetric thickness profile, International Journal of Mechanical Sciences 53 (2011) 827-838.

[10] D. Weisz-Patrault, A. Ehrlacher, N. Legrand, Non-linear simulation of coiling accounting for roughness of contacts and multiplicative elastic-plastic behavior, International Journal of Solids and Structures 94-95 (2016) 1-20.

[11] D. Weisz-Patrault, A. Ehrlacher, Imposed curvature of an elastic-plastic strip: application to simulation of coils, Mechanics \& Industry 18 (2017) 218-228.

[12] D. Weisz-Patrault, A. Ehrlacher, N. Legrand, E. Mathey, Non-linear numerical simulation of coiling by elastic finite strain model, Key Engineering Materials 651-653 (2015) 1060-1065.

[13] D. Weisz-Patrault, Coupled heat conduction and multiphase change problem accounting for thermal contact resistance, International Journal of Heat and Mass Transfer 104 (2017) 595-606.

[14] D. Weisz-Patrault, Multiphase model for transformation induced plasticity. extended leblond's model., Journal of the Mechanics and Physics of Solids 106 (2017) 152-175.

[15] M. Sutcliffe, Surface asperity deformation in metal forming processes, International Journal of Mechanical Sciences 30 (1988) 847-868.

[16] S. Sheu, W. R. Wilson, Mixed lubrication of strip rolling, Tribology transactions 37 (1994) $483-493$.

[17] J. Barber, Elasticity, volume 107 of Solid Mechanics and Its Applications, Springer, Berlin, 2003.

[18] N. Muskhelishvili, Some basic problems of the mathematical theory of elasticity, Noordhoff International Publishing, Groningen, 1953. 2nd edition (1977).

[19] D. Weisz-Patrault, S. Bock, K. Gürlebeck, Three-dimensional elasticity based on quaternion-valued potentials, International Journal of Solids and Structures 51 (2014) 3422-3430.

[20] J. Molleda, R. Usamentiaga, D. F. García, On-line flatness measurement in the steelmaking industry, Sensors 13 (2013) 10245-10272.

[21] D. Weisz-Patrault, Inverse cauchy method with conformal mapping : application to latent flatness defect detection during rolling process, International Journal of Solids and Structures 56 (2015) 175-193.

[22] J.-B. Leblond, J. Devaux, J. Devaux, Mathematical modelling of transformation plasticity in steels i: case of ideal-plastic phases, International journal of plasticity 5 (1989) 551-572.

[23] G. Watson, A treatise on the theory of bessel functions, Cambridge University Press, Cambridge, 1944.

[24] P. Papkovich, Solution générale des équations différentielles fondamentales d'élasticité, exprimée par trois fonctions harmoniques, CR Acad. Sci. Paris 195 (1932) 513-515.

[25] D. Weisz-Patrault, A. Ehrlacher, N. Legrand, Evaluation of contact stress during rolling process, by three dimensional analytical inverse method, International Journal of Solids and Structures 50 (2013) 3319-3331.

[26] CEA, Cast3m, 2011. Commissariat A l'Energie Atomique, http://www-cast3m.cea.fr/.

[27] M. Martinez, Jonction 16 MND 5-Inconel 690-316 LN par soudage-diffusion: élaboration et calcul des contraintes résiduelles de procédé, Ph.D. thesis, ENSMP, 1999.

[28] S. Grostabussiat-Petit, Conséquence mécanique des transformations structurales dans les alliages ferreux, Ph.D. thesis, INSA Lyon, 2000. 